\title{
A FAR-INFRARED SPECTROSCOPIC SURVEY OF INTERMEDIATE REDSHIFT (ULTRA) LUMINOUS INFRARED GALAXIES
}

\author{
Georgios E. Magdis ${ }^{1,2}$, D. Rigopoulou ${ }^{1,3}$, R. Hopwood ${ }^{4}$, J.-S. Huang ${ }^{5,6,7}$, D. Farrah ${ }^{8}$, C. PeArson $^{3,9,23}$, \\ Almudena Alonso-Herrero ${ }^{10}$, J. J. Bock ${ }^{11,12}$, D. Clements ${ }^{4}$, A. Cooray ${ }^{12,13}$, M. J. Griffin ${ }^{14}$, S. Oliver ${ }^{15}$,

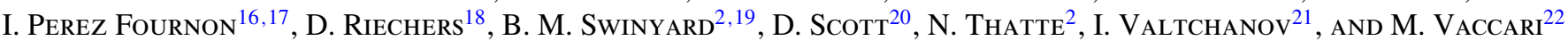 \\ ${ }^{1}$ Department of Physics, University of Oxford, Keble Road, Oxford OX1 3RH, UK \\ ${ }^{2}$ Institute for Astronomy, Astrophysics, Space Applications and Remote Sensing, National Observatory of Athens, GR-15236 Athens, Greece \\ ${ }^{3}$ RAL Space, Science, and Technology Facilities Council, Rutherford Appleton Laboratory, Didcot OX11 0QX, UK \\ ${ }^{4}$ Physics Department, Imperial College London, South Kensington Campus, London SW7 2AZ, UK \\ ${ }^{5}$ National Astronomical Observatories of China, Chinese Academy of Sciences, Beijing 100012, China \\ ${ }^{6}$ China-Chile Joint Center for Astronomy, Chinese Academy of Sciences, Camino El Observatorio, 1515, Las Condes, Santiago, Chile \\ ${ }^{7}$ Harvard-Smithsonian Center for Astrophysics, 60 Garden Street, Cambridge, MA 02138, USA \\ ${ }^{8}$ Department of Physics, Virginia Tech, Blacksburg, VA 24061, USA \\ ${ }^{9}$ The Open University, Milton Keynes MK7 6AA, UK \\ ${ }^{10}$ Instituto de Fisica de Cantabria, CSIC-UC, E-39006 Santander, Spain \\ ${ }^{11}$ California Institute of Technology, 1200 East California Boulevard, Pasadena, CA 91125, USA \\ 12 Jet Propulsion Laboratory, 4800 Oak Grove Drive, Pasadena, CA 91109, USA \\ ${ }^{13}$ Department of Physics and Astronomy, University of California, Irvine, CA 92697, USA \\ ${ }^{14}$ School of Physics and Astronomy, Cardiff University, Queens Buildings, The Parade, Cardiff CF24 3AA, UK \\ ${ }^{15}$ Astronomy Centre, Department of Physics and Astronomy, University of Sussex, Brighton BN1 9QH, UK \\ ${ }^{16}$ Instituto de Astrofsica de Canarias (IAC), 38200, La Laguna, Tenerife, Spain; ipf@iac.es \\ ${ }^{17}$ Departamento de Astrofsica, Universidad de La Laguna (ULL), E-38205 La Laguna, Tenerife, Spain \\ ${ }^{18}$ Department of Astronomy, Cornell University, 220 Space Sciences Building, Ithaca, NY 14853, USA \\ ${ }^{19}$ Department of Physics and Astronomy, University College London, Gower Street, London WC1E 6BT, UK \\ ${ }^{20}$ Department of Physics and Astronomy, University of British Columbia, 6224 Agricultural Road, Vancouver, BC V6T1Z1, Canada \\ ${ }^{21}$ Herschel Science Centre, European Space Astronomy Centre, Villanueva de la Canada, E-28691 Madrid, Spain \\ 22 Astrophysics Group, Physics Department, University of the Western Cape, Private Bag X17, 7535 Bellville, Cape Town, South Africa \\ Received 2014 June 18; accepted 2014 September 16; published 2014 November 5
}

\begin{abstract}
We present Herschel far-IR photometry and spectroscopy as well as ground-based CO observations of an intermediate redshift $(0.21 \leqslant z \leqslant 0.88)$ sample of Herschel-selected (ultra)-luminous infrared galaxies $\left(L_{\mathrm{IR}}>\right.$ $\left.10^{11.5} L_{\odot}\right)$. With these measurements, we trace the dust continuum, far-IR atomic line emission, in particular [C II $157.7 \mu \mathrm{m}$, as well as the molecular gas of $z \sim 0.3$ luminous and ultraluminous infrared galaxies (LIRGs and ULIRGs) and perform a detailed investigation of the interstellar medium of the population. We find that the majority of Herschel-selected intermediate redshift (U)LIRGs have $L_{\mathrm{CI}} / L_{\mathrm{FIR}}$ ratios that are a factor of about 10 higher than that of local ULIRGs and comparable to that of local normal and high- $z$ star-forming galaxies. Using our sample to bridge local and high- $z$ [C II] observations, we find that the majority of galaxies at all redshifts and all luminosities follow an $L_{\mathrm{C} \text { II }}-L_{\mathrm{FIR}}$ relation with a slope of unity, from which local ULIRGs and high- $z$ active-galactic-nucleus-dominated sources are clear outliers. We also confirm that the strong anti-correlation between the $L_{\mathrm{C} \text { II }} / L_{\mathrm{FIR}}$ ratio and the far-IR color $L_{60} / L_{100}$ observed in the local universe holds over a broad range of redshifts and luminosities, in the sense that warmer sources exhibit lower $L_{\mathrm{C}} / L_{\mathrm{FIR}}$ at any epoch. Intermediate redshift ULIRGs are also characterized by large molecular gas reservoirs and by lower star formation efficiencies compared to that of local ULIRGs. The high $L_{\mathrm{C} \text { II }} / L_{\mathrm{FIR}}$ ratios, the moderate star formation efficiencies $\left(L_{\mathrm{IR}} / L_{\mathrm{CO}}^{\prime}\right.$ or $L_{\mathrm{IR}} / M_{\mathrm{H}_{2}}$ ), and the relatively low dust temperatures of our sample (which are also common characteristics of high- $z$ star-forming galaxies with ULIRG-like luminosities) indicate that the evolution of the physical properties of (U)LIRGs between the present day and $z>1$ is already significant by $z \sim 0.3$.
\end{abstract}

Key words: galaxies: evolution - galaxies: star formation - gamma rays: ISM - infrared: galaxies infrared: general

Online-only material: color figures

\section{INTRODUCTION}

Luminous and ultraluminous infrared galaxies (LIRGs and ULIRGs) are the most powerful class of star-forming galaxies in the universe, with bolometric infrared luminosities $\left(L_{\mathrm{IR}}\right)$ in the range of $10^{11} L_{\odot}<L_{\mathrm{IR}}<10^{12} L_{\odot}$ and $>10^{12} L_{\odot}$, respectively, which correspond to star formation rates (SFRs) from tens to thousands of solar masses per year. While rare at the present day,

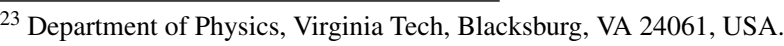

their comoving density increases by a factor of $\sim 1000$ as we move to higher redshifts and they account for more than $50 \%$ of the total SFR density in the universe at $z \sim 1-2$ (e.g., Le Floc'h et al. 2005; Magnelli et al. 2011; Murphy et al. 2011). As the hosts of the bulk of star formation activity during the epoch of galaxy assembly (at $z \sim 2$ ), placing (U)LIRGs $\left(L_{\mathrm{IR}}>10^{11} L_{\odot}\right)$ in the current framework of galaxy evolution is a cornerstone of modern astrophysics.

Besides the strong evolution in their number density, recent studies have pointed out some distinct differences in the 
properties of the present day and high- $z(z>1)$ ULIRGs. Local ULIRGs $(z<0.2)$ are almost always associated with a disturbed system of galaxies undergoing mergers (e.g., Armus et al. 1987; Melnick \& Mirabel 1990; Clements et al. 1996; Farrah et al. 2001; Bushouse et al. 2002), are predominantly sources with warm spectral energy distributions (SEDs; dust temperature, $T_{\mathrm{d}}$ exceeding, on average, $40 \mathrm{~K}$; e.g., Clements et al. 2010; Symeonidis et al. 2011), high star formation efficiencies (SFEs; defined as the ratio of the total infrared luminosity over the molecular gas mass, $\mathrm{SFE}=L_{\mathrm{IR}} / M_{\mathrm{H}_{2}}>100 L_{\odot} / M_{\odot}$; e.g., Solomon et al. 1992; Gao \& Solomon 2004), exhibit a general deficiency of far-IR atomic lines with respect to local normal galaxies (e.g., Luhman et al. 2003; Graciá-Carpio et al. 2011; Farrah et al. 2013; Diaz-Santos et al. 2013), and are characterized by compact star-forming regions, confined within the central kiloparsec of the merging systems (e.g., Solomon et al. 1997; Bryant \& Scoville 1999; Soifer et al. 2000).

However, there is strong evidence that the aforementioned properties are not representative of the ULIRG population in the early universe. For example, morphological studies have shown that high- $z$ ULIRGs appear to be a mixture of merging/ interacting systems and disk galaxies (e.g., Kartaltepe et al. 2012; Kaviraj et al. 2013). Furthermore, studies initiated with the Spitzer Space Telescope (Spitzer; Werner et al. 2004) and more recently with the Herschel Space Observatory (Herschel; Pilbratt et al. 2010) have shown that their infrared SEDs are more similar to those of local galaxies of lower luminosities, exhibiting stronger polycyclic aromatic hydrocarbon (PAH) features (e.g., Farrah et al. 2008; Pope et al. 2006) and colder dust temperatures (e.g., Pope et al. 2006, 2008; Farrah et al. 2008; Lonsdale et al. 2009; Symeonidis et al. 2009, 2013; Muzzin et al. 2010; Magdis et al. 2010, 2012b; Elbaz et al. 2011) than local ULIRGs. Observations of the carbon monoxide molecule (CO) have also revealed an extended distribution and ordered rotation of the molecular gas $\left(M_{\text {gas }}\right)$, as well as moderate SFEs that are a factor of 10 lower compared to that of local ULIRGs (e.g., Genzel et al. 2010; Daddi et al. 2010a, 2010b; Magdis et al. $2012 b$ ). Hints of an increase in the physical scale of ULIRGs at high- $z$ have also been provided by radio and mid-IR studies, suggesting that, on average, local ULIRGs are five times smaller (e.g., Rujopakarn et al. 2011; Elbaz et al. 2011). Finally, far-IR spectroscopy targeting the [C II] $157.7 \mu \mathrm{m}$ atomic line of high- $z$ star-formation-dominated ULIRGs $(z>1)$ revealed [C II]-to-far infrared luminosity ratios $\left(L_{\mathrm{C} \text { II }} / L_{\mathrm{FIR}}\right)$ similar to local star-forming galaxies, and much above the median values found for local ULIRGs, suggestive of softer radiation fields and more extended star-forming regions (e.g., Ivison et al. 2010b; Hailey-Dunsheath et al. 2010; Stacey et al. 2010; Valtchanov et al. 2011).

While a complete picture has not yet emerged, it is established that there has been a strong evolution in the physical properties and possibly of the mechanism (i.e., mergers versus secular evolution) that drives the star formation activity in ULIRGs between the present day and $z>1$. To a great extent, these observations also suggest that a simple luminosity classification is perhaps only meaningful at a given cosmic epoch and cannot be used to draw analogues between the present day and the early universe galaxy populations. However, ULIRGs have so far only been extensively studied either in the local universe $z<0.2$ or at high- $z(z>1)$. We are therefore building the picture at the two ends of their evolution while missing the critical intermediate epoch of $0.2<z<1.0$. To fully understand the evolution of the ULIRG phenomenon, which is responsible for the bulk of star formation in the universe, we need to sample the evolution in the $0.2<z<1$ redshift range, an era when the universe experienced a strong increase in its SFR density.

To bridge the gap between local and high- $z$ ULIRGs, here we perform a detailed and in depth investigation of a sample of 17 Herschel-selected (U)LIRGs in the redshift range of $0.21<$ $z<0.8$, with fully characterized infrared SEDs from Spitzer and Herschel. Using the Spectral and Photometric Imaging REceiver-Fourier Transform Spectrometer (Griffin et al. 2010) on board Herschel we target primarily the [C II] $157.7 \mu \mathrm{m}$ emission line, as well as the [N II] $205 \mu \mathrm{m}$ and [O I] $145 \mu \mathrm{m}$ atomic lines. These lines, and in particular [C II] $157.7 \mu \mathrm{m}$, are important coolants of the interstellar medium (ISM) and can serve as powerful tracers of its physical conditions. As they are rapidly becoming the benchmark for the study of the cool atomic gas in local and distant $(z>1)$ galaxies, our survey, for the first time, offers the opportunity to study these lines, and therefore the properties of the ISM, in intermediate redshift galaxies. We note that the current sample, first presented in Rigopoulou et al. (2014), is the only existing sample of (U)LIRGs in this redshift range with [C II] measurements. Our analysis is also complemented by follow-up, ground-based $\mathrm{CO}$ observations that trace the molecular gas of the sources, resulting in a unique sample of ULIRGs with far-IR spectroscopic and multiwavelength photometric data, which is ideal for exploring the evolution of ULIRGs.

This paper is organized as follows. In Sections 2 and 3, we present the selection criteria of the sample and the subsequent observations and data analysis, respectively. In Section 4, we derive the infrared properties of the sample, based on SED fitting, and in Section 5 we explore various correlations between the strength of the [C II] emission relative to the far-IR infrared luminosity and other parameters, such the total far infrared luminosity, the dust temperature, and the SFE of galaxies across the cosmic time. In Section 6, we discuss the implications of our findings on the evolution of the (U)LIRGs phenomenon, while a summary of our results is given in Section 7. Throughout this article, we adopt the WMAP-7 cosmology $\left(\Omega_{\mathrm{m}}=0.273\right.$, $\Omega_{\Lambda}+\Omega_{\mathrm{m}}=1, \Omega_{\mathrm{k}}=0$ and $H_{0}=70.4 \mathrm{~km} \mathrm{~s}^{-1} \mathrm{Mpc}^{-1}$; Larson et al. 2011).

\section{THE SAMPLE}

We selected targets to investigate the properties of the ISM of intermediate redshift (U)LIRGs through the detection of far-IR atomic lines, especially [C II], [N II], and [OII]. The two main criteria were as follows. First, a lower limit on the brightness in the far-IR to ensure the detection of the source against the background emission of the telescope $(\sim 80 \mathrm{~K})$ and the uncertainties in the instrument and telescope model (see the SPIRE-FTS handbook). Second, the redshift range to ensure that the redshifted [C II] emission falls within the spectral bandwidth of Fourier transform spectroscope (FTS; i.e., $z>0.21)$. The starting point for our target selection was the Herschel-SPIRE photometric catalogs from the Herschel Multi-tiered Extragalactic Survey (Oliver et al. 2012). The Herschel Multi-Tiered Extragalactic Survey (HerMES) mapped at $250 \mu \mathrm{m}, 350 \mu \mathrm{m}$, and $500 \mu \mathrm{m}$ some of the most well studied fields in the sky, including Bootes, EGS, COSMOS, and HDFN. Photometric catalogs were produced using both a blind and a $24 \mu \mathrm{m}$ prior based source extraction technique (Roseboom et al. 2010). Using the prior based catalogs, we searched for sources with $S_{250}>150 \mathrm{mJy}$ in all fields covered by HerMES, producing a master $250 \mu \mathrm{m}$ target list (see Section 3.1). As stated 
Table 1

Sample Definition

\begin{tabular}{|c|c|c|c|c|c|}
\hline Source & $\begin{array}{l}\text { R.A. }^{a} \\
(\mathrm{~J} 2000)\end{array}$ & $\begin{array}{c}\text { Decl. } \\
(\mathrm{J} 2000)\end{array}$ & $z_{\text {spec }}$ & Reference $^{b}$ & Type $^{c}$ \\
\hline CDFS2 & $03: 28: 18.0$ & $-27: 43: 08.0$ & 0.248 & Optical(1) & $\mathrm{AGN}(1)$ \\
\hline BOOTES3 & $14: 28: 49.7$ & $+34: 32: 40.3$ & 0.219 & $\operatorname{IRS}(2)$ & $\mathrm{SB}(2)$ \\
\hline BOOTES2 & $14: 32: 34.9$ & $+33: 28: 32.3$ & 0.250 & $\operatorname{IRS}(2,8)$ & $\mathrm{SB}(8)$ \\
\hline SWIRE4 & $10: 32: 37.4$ & $+58: 08: 46.0$ & 0.251 & $\operatorname{IRS}(3)$ & $\mathrm{SB}(3)$ \\
\hline ELAISS & $00: 40: 14.6$ & $-43: 20: 10.1$ & 0.265 & Optical(4) & $\mathrm{SB}(4)$ \\
\hline CDFS1 & $03: 29: 04.3$ & $-28: 47: 52.9$ & 0.289 & Optical(5) & $\cdots$ \\
\hline BOOTES1 & $14: 36: 31.9$ & $+34: 38: 29.1$ & 0.354 & $\operatorname{IRS}(6)$ & $\operatorname{AGN}(6)$ \\
\hline SWIRE2 & $10: 51: 13.4$ & $+57: 14: 26.2$ & 0.362 & Optical(7) & $\cdots$ \\
\hline SWIRE5 & $10: 35: 57.9$ & $+58: 58: 46.2$ & 0.366 & $\mathrm{CO},[\mathrm{C}$ II $](8)$ & $\cdots$ \\
\hline FLS01 & $17: 20: 17.1$ & $+59: 16: 37.7$ & 0.417 & Optical(9), IRS(10) & Composite(10) \\
\hline FLS02 & $17: 13: 31.5$ & $+58: 58: 04.2$ & 0.436 & Optical-SWIFT(8), IRS(8) & $\mathrm{AGN}(8)$ \\
\hline SWIRE1 & $10: 47: 53.3$ & $+58: 21: 06.2$ & 0.887 & Optical(11) & QSO(11) \\
\hline $\mathrm{XMM}^{\mathrm{d}}$ & $02: 20: 16.5$ & $-06: 01: 43.2$ & 2.308 & (12) & SB \\
\hline HLSW-01 (SWIRE6) & $10: 57: 50.4$ & $+57: 30: 28.3$ & 2.957 & $\mathrm{CO}(13)$ & $\cdots$ \\
\hline ELAISN & $16: 10: 27.0$ & $+53: 12: 43.0$ & 0.693 & $z_{\text {phot }}$ & $\cdots$ \\
\hline SWIRE7 & 11:02:05.7 & $+57: 57: 40.6$ & 0.550 & $z_{\text {phot }}$ & $\cdots$ \\
\hline XMM2 & $02: 19: 57.3$ & $-05: 23: 48.9$ & 0.280 & $z_{\text {phot }}$ & $\cdots$ \\
\hline
\end{tabular}

Notes.

${ }^{\text {a }}$ Coordinates from MIPS $24 \mu \mathrm{m}$ continuum emission.

b Origin of the spectroscopic redshift: (1) Eales et al. (2009); (2) Weedman \& Houck (2008); (3) Magdis et al. (2013); (4) La Franca et al. (2004); (5) Norris et al. (2006); (6) Houck et al. (2007); (7) Oyabu et al. (2005); (8) This study; (9) Papovich et al. (2006); (10) Dasyra et al. (2009); (11) C. Gruppioni et al. 2013, private communication; (12) Fu et al. (2013); and (13) Scott et al. (2011).

c Source classification.

${ }^{\mathrm{d}}$ In the original sample, selection XMM1 was erroneously assigned a redshift of 0.502 , which corresponds to the foreground galaxy of the lensed SMGs HXMM01 (Fu et al. 2013).

e In the original sample selection, SWIRE6 was erroneously assigned a redshift of 0.584 , which corresponds to the foreground galaxy of the lensed HLSW-01 (Scott et al. 2011).

above, the flux cut was imposed by the telescope specifications as well as our desire to study far-IR luminous sources $\left(L_{\mathrm{IR}}>\right.$ $\left.10^{11} L_{\odot}\right) .^{24}$

Among the $250 \mu \mathrm{m}$ selected targets, 14 had spectroscopic redshifts with $0.21<z_{\text {spec }}<0.9$, a redshift range where [C II] $157.7 \mu \mathrm{m}$ and [N II] $205 \mu \mathrm{m}$ emission is covered by FTS. The initial spectroscopic sample was further supplemented with the three brightest sources at $250 \mu \mathrm{m}$ and $z_{\text {phot }}>0.3$ resulting in a final "[C $\mathrm{II}]$-sample" of 17 galaxies. No other criteria (e.g., optical color, stellar mass, existence of an active galactic nucleus, AGN) were imposed. The final sample consists of 15 sources in the redshift range of $0.219 \leqslant z \leqslant 0.887$, and 2 sources, XMM1 and SWIRE6 at $z_{\text {spec }}=2.308$ and $z_{\text {spec }}=$ 2.957 , respectively. The latter were initially selected as the $z_{\text {spec }}=0.502$ and $z_{\text {spec }}=0.584$ foreground galaxies of the lensed SMGs HXMM01 (Fu et al. 2013) and HLSW-01 (Scott et al. 2011; Conley et al. 2011; Gavazzi et al. 2011; Riechers et al. 2011), respectively. The FTS spectra of these two lensed systems will be presented in detail in two companion papers. For the rest of the sources, the spectroscopic redshifts are drawn from the literature and are based on either optical or mid-IR spectra (see Table 1). Where available, optical line ratios (e.g., [N II]/ $\mathrm{H} \alpha$ ), the equivalent width (EW) of the $6.2 \mu \mathrm{m}$ PAH feature (e.g., Spoon et al. 2007), or the mid-IR color based on IRAC photometry (e.g., Donley et al. 2012) were also used to identify four AGNs among the galaxies in our sample (BOOTES1, SWIRE1, CDFS2, and FLS02). In total, we have 17 sources, of which 14 have spectroscopic and 3 have photometric redshifts.

\footnotetext{
24 Using various templates (M82, Arp220; Chary \& Elbaz 2001), we estimate a total infrared luminosity of $\log \left(L_{\mathrm{IR}} / L_{\odot}\right)>11.0$ for sources with $S_{250}=150 \mathrm{mJy}$ in the redshift range of $z=0.21-0.8$.
}

The median redshift of the sample, excluding the two high- $z$ lensed systems, is 0.351 . The full sample is presented in Table 1.

\section{OBSERVATIONS AND DATA ANALYSIS}

\subsection{Herschel and Ancillary Multi-wavelength Photometry}

We used Herschel-SPIRE observations of the fields ELAISN1, ELAIS-S1, Lockman Hole, XMM, Bootes, CDFS, and FLS, obtained as part of HerMES (Oliver et al. 2012). For our sources, we employed the photometric catalogs at 250, 350 , and $500 \mu \mathrm{m}$ that were produced for each field by using a prior source extraction, guided by the position of known $24 \mu \mathrm{m}$ sources. An extensive description of the cross-identification prior source extraction (XID) method is given in Roseboom et al. $(2010,2012)$. The main advantage of this method is that reliable fluxes can be extracted close to the formal $\approx 4-5 \mathrm{mJy}$ SPIRE confusion noise (Nguyen et al. 2010) by estimating the flux contributions from nearby sources within one beam. The $24 \mu \mathrm{m}$ prior positional information reduces the impact of confusion noise and so the approximate $3 \sigma$ limit for the SPIRE catalog at $250 \mu \mathrm{m}$ becomes $\approx 9-15 \mathrm{mJy}$. The drawback of this technique is that the resulting catalogs could be missing sources without a $24 \mu \mathrm{m}$ counterpart, that is, $24 \mu \mathrm{m}$ dropouts (see Magdis et al. 2011). All sources were originally selected to have $S_{250}>150 \mathrm{mJy}$, although a subsequent deblending analysis of FLS01 yielded a flux density of $S_{250}=80.6 \mathrm{mJy}$.

Our targets also benefit from ancillary Spitzer MIPS (24, 70, and $160 \mu \mathrm{m})$ and $\operatorname{IRAC}(3.6,4.5,5.8$, and $8.0 \mu \mathrm{m})$ observations. Finally, six of our sources have been followed up with Infrared Spectrograph (IRS) spectroscopy. The IRS reduced spectra 
Table 2

Far-IR Photometry

\begin{tabular}{|c|c|c|c|c|c|c|}
\hline Source & $\begin{array}{c}S_{24} \\
(\mathrm{mJy})\end{array}$ & $\begin{array}{c}S_{70} \\
(\mathrm{mJy})\end{array}$ & $\begin{array}{c}S_{160} \\
(\mathrm{mJy})\end{array}$ & $\begin{array}{l}S_{250}{ }^{\mathrm{a}} \\
(\mathrm{mJy})\end{array}$ & $\begin{array}{c}S_{350} \\
(\mathrm{mJy})\end{array}$ & $\begin{array}{c}S_{500} \\
(\mathrm{mJy})\end{array}$ \\
\hline BOOTES1 & $52.2 \pm 0.2$ & $614.0 \pm 1.5$ & $605.5 \pm 3.4$ & $367.4 \pm 2.1$ & $161.1 \pm 2.3$ & $58.5 \pm 3.4$ \\
\hline BOOTES2 & $4.3 \pm 0.2$ & $347.0 \pm 1.0$ & $294.5 \pm 2.5$ & $175.9 \pm 1.9$ & $69.3 \pm 2.1$ & $25.1 \pm 2.2$ \\
\hline BOOTES3 & $8.7 \pm 0.4$ & $244.0 \pm 0.8$ & $518.8 \pm 3.7$ & $351.4 \pm 1.9$ & $164.1 \pm 1.9$ & $57.2 \pm 2.3$ \\
\hline CDFS1 & $2.9 \pm 0.1$ & $93.0 \pm 1.6$ & $355.9 \pm 5.9$ & $195.4 \pm 2.5$ & $85.7 \pm 3.6$ & $41.3 \pm 12.5$ \\
\hline CDFS2 & $5.0 \pm 0.1$ & $199.2 \pm 2.2$ & $371.8 \pm 6.7$ & $227.6 \pm 2.5$ & $100.9 \pm 2.1$ & $33.1 \pm 2.5$ \\
\hline ELAISS & $3.9 \pm 0.4$ & $56.4 \pm 2.1$ & $201.3 \pm 10.6$ & $158.9 \pm 2.3$ & $78.3 \pm 2.7$ & $28.3 \pm 3.0$ \\
\hline FLS01 & $3.3 \pm 0.4$ & $65.0 \pm 0.3$ & $\ldots$ & $80.6 \pm 2.4$ & $29.6 \pm 6.0$ & $\cdots$ \\
\hline FLS02 & $6.2 \pm 0.7$ & $230.1 \pm 0.3$ & $314.7 \pm 6.1$ & $172.2 \pm 2.4$ & $90.5 \pm 2.4$ & $38.8 \pm 3.4$ \\
\hline SWIRE1 & $5.2 \pm 0.2$ & $78.0 \pm 1.4$ & $279.2 \pm 6.2$ & $199.8 \pm 4.0$ & $139.9 \pm 4.8$ & $45.1 \pm 13.6$ \\
\hline SWIRE2 & $2.7 \pm 0.04$ & $42.7 \pm 1.1$ & $241.74 \pm 4.9$ & $201.5 \pm 4.2$ & $148.3 \pm 4.4$ & $69.1 \pm 5.5$ \\
\hline SWIRE4 & $6.1 \pm 0.5$ & $139.2 \pm 1.8$ & $547.0 \pm 6.7$ & $173.1 \pm 7.3$ & $54.8 \pm 6.4$ & $14.6 \pm 7.9$ \\
\hline SWIRE5 & $5.9 \pm 0.4$ & $96.0 \pm 1.8$ & $303.2 \pm 8.1$ & $174.1 \pm 4.0$ & $83.4 \pm 4.1$ & $45.7 \pm 6.1$ \\
\hline HLSW-01 (SWIRE6) & $5.5 \pm 0.4$ & $22.2 \pm 3.0$ & $\cdots$ & $425.0 \pm 10.0$ & $340.0 \pm 10.0$ & $233.0 \pm 10.0$ \\
\hline $\mathrm{XMM} 1^{\mathrm{b}}$ & $2.2 \pm 0.10$ & $7.9 \pm 3.8$ & $102.1 \pm 6.0$ & $180.3 \pm 14.3$ & $192.1 \pm 15.5$ & $131.6 \pm 11.3$ \\
\hline ELAISN & $3.8 \pm 0.4$ & $\ldots$ & $245.9 \pm 7.2$ & $149.2 \pm 1.3$ & $83.6 \pm 1.2$ & $23.7 \pm 1.4$ \\
\hline SWIRE7 & $3.3 \pm 0.4$ & $72.4 \pm 1.3$ & $224.5 \pm 4.9$ & $191.2 \pm 4.9$ & $98.3 \pm 4.1$ & $41.7 \pm 4.9$ \\
\hline XMM2 & $3.8 \pm 0.2$ & $336.0 \pm 6.0$ & $481.2 \pm 11.2$ & $188.7 \pm 2.35$ & $86.3 \pm 5.5$ & $<15$ \\
\hline
\end{tabular}

Notes.

a The quoted uncertainties in the SPIRE photometry here do not take into account the confusion noise.

b Extensive photometry for XMM1 can be found in Fu et al. (2013). Here the quoted flux density at $160 \mu \mathrm{m}$ corresponds to the PACS measurement, in contrast to the rest of the sources, which corresponds to flux densities as measured by MIPS.

were downloaded from CASSIS.v6 $6^{25}$ (Lebouteiller et al. 2011) and PAH luminosities and EWs were measured using PAHFIT (Smith et al. 2007). The MIPS and SPIRE photometry for our sample is summarized in Table 2.

\subsection{FTS Observations and Data Analysis}

The (U)LIRGs in our sample were observed with the SPIRE FTS on board Herschel between 2012 March and 2013 January. The FTS observed 100 repetitions $(13,320$ seconds total integration time) on each target with sparse spatial sampling, in high spectral resolution $\left(0.048 \mathrm{~cm}^{-1}\right)$ mode. The SPIRE FTS measures the Fourier transform of the spectrum of a source using two bolometer detector arrays, simultaneously covering wavelength bands of 194-313 $\mu \mathrm{m}$ (SSW) and 303-671 $\mu \mathrm{m}$ (SLW).

The data were reduced with the user pipeline ( $T$. Fulton et al. 2014, in preparation) in HIPE version 11, or version 12.1 if the slightly wider frequency bands proved advantageous for fitting lines near the frequency band edges. All ULIRGs in our sample are extremely faint targets for the FTS, with continua $\ll 1 \mathrm{Jy}$, and therefore require processing beyond a standard reduction.

For those observations taken within the first eight hours of an FTS pair of observing days, a correction was made to the telescope model subtracted within the pipeline. This adjustment is necessary due to low detector temperatures during the SPIRE cooler recycle, which is not fully accounted for by the nonlinearity correction for certain detectors, and therefore the spectra fail to be properly calibrated. The empirical correction applied is based on the linear correlation of detector temperature and SLW flux density of point-source-calibrated spectra of dark sky observations (see Swinyard et al. 2014 for details). For correctly calibrated data, this relationship is flat.

A bespoke relative spectral response function (RSRF), constructed from selected long dark sky observations, was applied if found to improve the noise in the point source calibrated

\footnotetext{
25 http://cassis.sirtf.com/atlas/
}

product. However, for most observations, the standard RSRF was used, which was constructed using the scans of all dark sky observations. Once reduced to the point-source-calibrated stage, the main issue affecting FTS observations of faint compact sources is imperfect subtraction of the telescope RSRF and, for the low-frequency end of SLW, the instrument RSRF. To correct the distortions introduced by the point source calibration of this extended telescope residual, a background subtraction was performed. For FTS observations of point sources, the target is positioned in the center of the SSW detector, which overlaps with the central SLW detector. Both detector arrays are set out in a closely packed hexagonal pattern, which essentially provides concentric rings of off-axis detectors around the respective center detector (see Swinyard et al. 2014 for the precise layout). The first ring of SLW detectors and the first and second rings for SSW were smoothed to remove small-scale noise and inspected to reject any outliers due to clipping before averaging the selected spectra. This average gives a measure of the residual background, which was subtracted from the respective on-axis detectors.

The optimal reduction spectra were examined for spectral features at the positions of expected lines. Due to the faint nature of the targets, many of the lines are $<5 \sigma$, and since lowfrequency noise in an FTS spectrum can easily mimic a faint line of similar significance, two checks were performed for reliability and to gain statistically robust line measurements, both utilizing the un-averaged point-source-calibrated data. These two methods are described in R. Hopwood et al. (2014, in preparation). First, a Jackknife technique was used to minimize the chance of spurious detections. Each un-averaged set of 200 scans was divided into groups of sequential subsets. An average was taken for each subset, and this repeated for subsets of decreasing number, i.e., 2 sets of 100 scans, then 4 sets of 50 scans, down to spectra averaged from 10 scan subsets. In order to assess the realness of a potential detection, the results were plotted for a visual comparison of each averaged spectra in a subset and across subset size. Spectra of 10 scans should present 


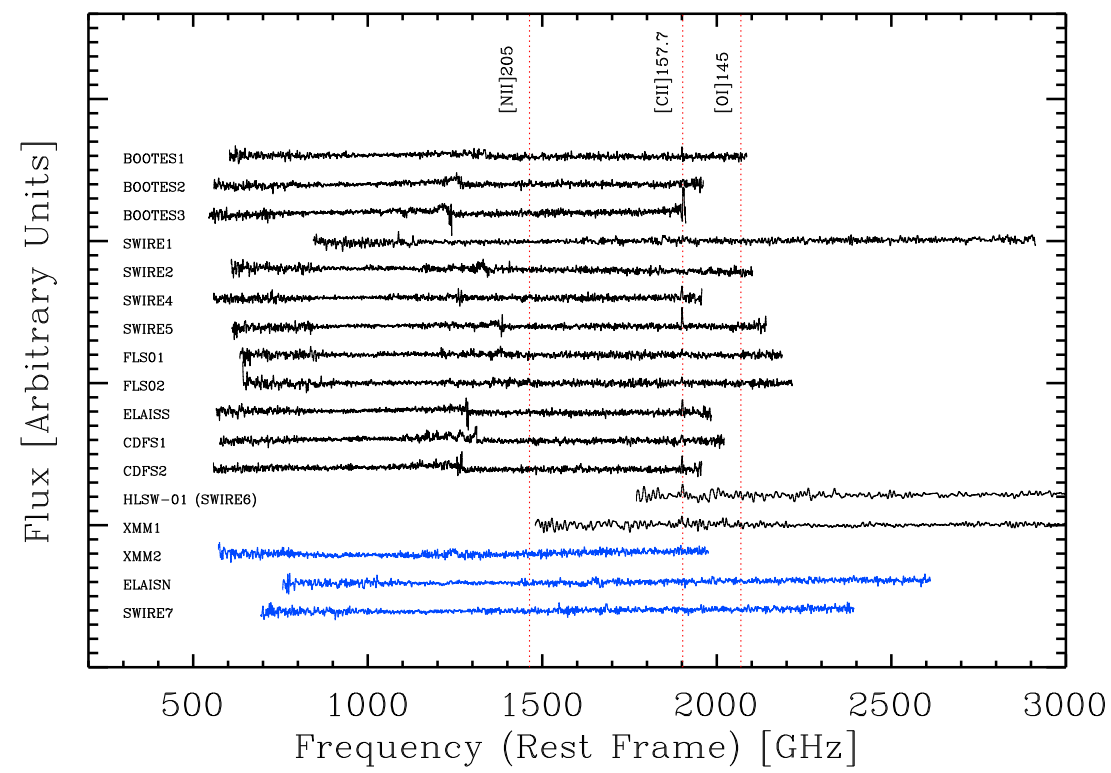

Figure 1. Spectroscopic Herschel-FTS atlas of the sources in our sample showing the full rest-frame spectra in arbitrary units. The vertical lines correspond to the frequencies of the [N II] $205 \mu \mathrm{m}$, [C II] $157.7 \mu \mathrm{m}$, and [O I] $145 \mu \mathrm{m}$ atomic lines. The blue spectra correspond to sources with photometric redshift.

(A color version of this figure is available in the online journal.)

as random noise unless there is high systematic noise, which can manifest as a detection in the fully averaged spectrum. The comparison of spectra averaged from 20 scans or more should start to show a consistent peak at the line position for all subsets, while the nearby data should still present as more random, with peaks of less significance.

Second, a bootstrap method was used to measure the line flux for any line assessed as potentially real and to provide a background level, which adds a second reliability check. For any given observation, the un-averaged scan set was randomly sampled until the number in the parent population (of 200) was reached. These random scans were then averaged and line measurements taken using the same basic fitting technique as for a standard average spectrum. The instrumental line shape of the FTS is well approximated by a sinc function (see Swinyard et al. 2014, R. Hopwood et al. 2014, in preparation for more details). Therefore, for unresolved lines, sinc functions were fitted simultaneously with a polynomial of the order of three for the continuum. Several of the detected [C II] lines were found to be partially resolved and so a sinc convolved with a Gaussian was fitted in these cases, using the sincGAuss function within HIPE. When bootstrapping the line measurements, a more aggressive baseline subtraction was performed to each scan prior to resampling. A set of random frequency positions was also generated and the same line fitting process applied. The resampling and fitting was repeated 10,000 times for each observation and a Gaussian fitted to the resulting line flux distribution to obtain the mean line flux. The Gaussian width was taken as the associated $1 \sigma$ uncertainties. The distributions obtained from the set of random frequency positions establishes a background level, above which a real spectral feature is strongly suggested. This level was generally $\sim 2 \sigma$, so for any line found to be at $<2 \sigma$, the bootstrapped flux density is taken as an upper limit, but only if the detection is supported by the Jackknife and other visual checks.

The full extracted spectra are presented in Figure 1 while in Figure 2 we show the detected lines along with the bestfit model. The derived flux densities, line widths, luminosities, and $3 \sigma$ upper limits for the undetected lines are summarized in
Table 3. In total, we detect [C II] 157.7 emission from 12 out of the 14 sources in our spectroscopic sample. The [O I] 145 line is also detected in one of our sources (SWIRE5), while [N II] 205, remains undetected for the entire sample. For the three sources with photometric redshifts, we do not detect any lines at the expected frequencies or by letting the redshift vary as a free parameter in the fitting process. In Table 3, we provide the upper limits of the lines measured at the expected frequencies based on their photometric redshifts. However, since the actual redshift of these sources remains largely unconstrained, we choose to omit them from the subsequent analysis.

\subsection{CO Observations and Data Analysis}

We carried out follow-up, CO single-dish observations for nine galaxies in our sample. We used the Eight MIxer Receiver (EMIR; Carter et al. 2012) on the IRAM $30 \mathrm{~m}$ telescope at Pico Veleta, Spain, in 2012 June for six northern sources and the Swedish Heterodyne Facility Instrument (Vassilev et al. 2008) on the Atacama Pathfinder EXperiment ${ }^{26}$ (APEX; Güsten et al. 2006) $12 \mathrm{~m}$ telescope from 2012 August to October (run ID 090.B-0708A) and from 2013 April to July (run ID 091.B-0312A) for three southern sources (CDFS1, CDFS2, and ELAISS).

IRAM observations were carried out at $1.2,2.0$, and $3.0 \mathrm{~mm}$ (E0, E1, and E2 bands of EMIR), primarily targeting the $\mathrm{CO}[1-0]$ emission $\left(v_{0}=115.271 \mathrm{GHz}\right)$ line from our galaxies. If $\mathrm{CO}[1-0]$ was not accessible, we searched for $\mathrm{CO}[2-1]$ $\left(v_{0}=230.538 \mathrm{GHz}\right)$ or $\mathrm{CO}[3-2]\left(v_{0}=345.796 \mathrm{GHz}\right)$. When possible, we carried out simultaneous observations of two lines using $\mathrm{E} 0 / \mathrm{E} 1(\mathrm{CO}[1-0], \mathrm{CO}[2-1])$ or $\mathrm{E} 1 / \mathrm{E} 2(\mathrm{CO}[2-1]$, $\mathrm{CO}[3-2])$ EMIR band combinations. The broadband EMIR receivers were tuned in single sideband mode, with a total bandwidth of $4 \mathrm{GHz}$ per polarization which covers a velocity range of around $12,000 \mathrm{~km} \mathrm{~s}^{-1} 1$ at $3 \mathrm{~mm}, 8000 \mathrm{~km} \mathrm{~s}^{-1}$ at $2 \mathrm{~mm}$,

\footnotetext{
26 This publication is based on data acquired with the Atacama Pathfinder Experiment (APEX). APEX is a collaboration between the Max-PlanckInstitut fur Radioastronomie, the European Southern Observatory, and the Onsala Space Observatory.
} 

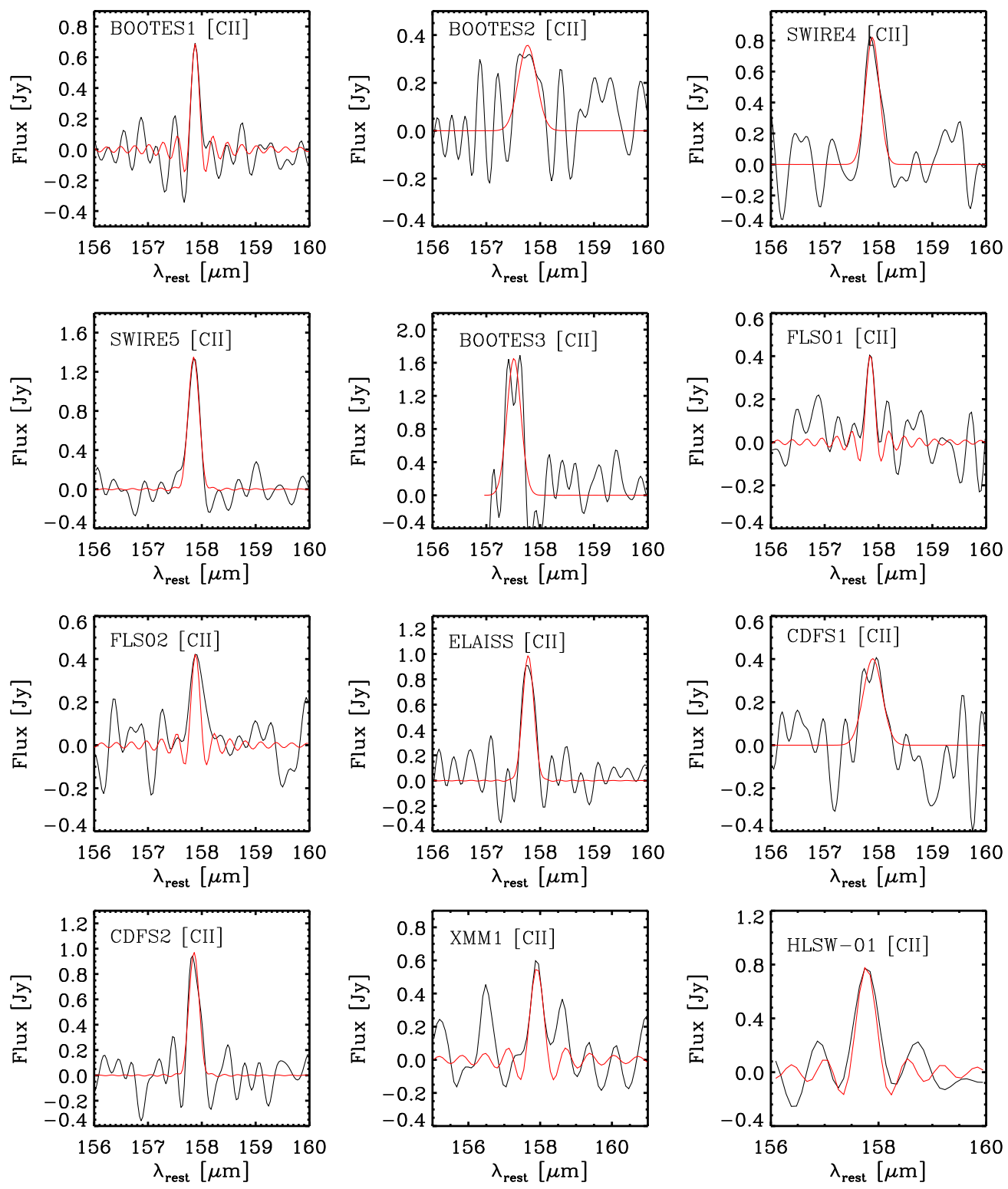

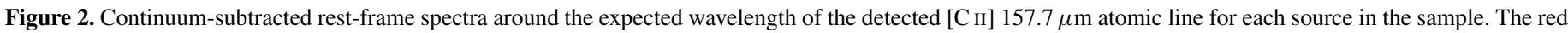
curves correspond to the best-fit model, which was used to extract the emission line fluxes and widths (see Section 3.2).

(A color version of this figure is available in the online journal.)

and $\sim 5000 \mathrm{~km} \mathrm{~s}^{-1}$ at $1.2 \mathrm{~mm}$. The observations were carried out in wobbler-switching mode, with reference positions offset by $2^{\prime}$ in azimuth.

We spent two to five hours on each galaxy, resulting in a noise level of $0.5-1.5 \mathrm{mK}$ per $30 \mathrm{~km} \mathrm{~s}^{-1}$ channel for all sources. The system temperatures were relatively stable with an average of $\sim 100 \mathrm{~K}$ at $3 \mathrm{~mm}, \sim 280 \mathrm{~K}$ at $2 \mathrm{~mm}$, and $\sim 300 \mathrm{~K}$ at $1.2 \mathrm{~m}$, in $T_{\mathrm{A}}^{*}$. The pointing was regularly checked on continuum sources and yielded an accuracy of $3^{\prime \prime} \mathrm{rms}$. The temperature scale used is in main beam temperature, $T_{\mathrm{mb}}$. At $3 \mathrm{~mm}, 2 \mathrm{~mm}$, and $1.2 \mathrm{~mm}$, the telescope half-power beam width is $27^{\prime \prime}, 17^{\prime \prime}$, and $10^{\prime \prime}$, respectively, and the main beam efficiencies $\left(\eta_{\mathrm{mb}}=T_{\mathrm{A}}^{*} / T_{\mathrm{mb}}\right)$ are $0.85,0.70$, and 0.64 , respectively, with $S / T_{\mathrm{mb}}=4.8 \mathrm{Jy} \mathrm{K}^{-1}$ for all bands.

APEX observations were carried out in average weather conditions with precipitable water vapor $0.4<\mathrm{PWV}<1.5$ and in wobbler-switching mode, with a symmetrical azimuthal throw of $20^{\prime \prime}$ and a frequency of $0.5 \mathrm{~Hz}$. Pointing was checked every hour and found to be better than $3^{\prime \prime}$ (with a beam size of $20^{\prime \prime}$ ). The focus was checked on the available planets, especially after sunrise when the telescope deformations are largest. The on-source integration time was $\sim 2 \mathrm{hr}$ for CDFS 2 and ELAISS and $\sim 8 \mathrm{hr}$ for CDFS1, targeting the $\mathrm{CO}[3-2]$ emission line in all sources using APEX1 and APEX2 $(214-275 \mathrm{GHz}$ and $267-378 \mathrm{GHz}$, respectively). The achieved rms was around $0.4 \mathrm{mK}$ for CDFS1 and $0.9 \mathrm{mK}$ for the other two sources.

Both sets of spectra (APEX and IRAM) were reduced using CLASS (within the GILDAS-IRAM package). In short, each spectrum was averaged and reduced using linear baselines, and then binned to $50-60 \mathrm{~km} \mathrm{~s}^{-1}$. To determine the properties of the emission lines (observed flux, velocity centroid, and FWHM), we fitted Gaussian functions to the observed spectra. We obtained fits for all galaxies with a single Gaussian and with two Gaussian functions (in this case fixing the FWHM in each component to the same value). For all sources, a single Gaussian provided the best fit to the data, although in some 
Table 3

FTS Spectroscopy

\begin{tabular}{|c|c|c|c|c|c|c|c|}
\hline Source & $\begin{array}{c}F_{\left[\mathrm{O}_{\mathrm{I}]}\right] 145^{\mathrm{a}}} \\
\left(10^{-18} \mathrm{~W} \mathrm{~m}^{-2}\right)\end{array}$ & $\begin{array}{c}\log L_{\left[\mathrm{O}_{\mathrm{I}}\right] 145^{\mathrm{b}}} \\
\left(L_{\odot}\right)\end{array}$ & $\begin{array}{c}F_{[\mathrm{C} \mathrm{II}]} \\
\left(10^{-18} \mathrm{~W} \mathrm{~m}^{-2}\right)\end{array}$ & $\begin{array}{l}\text { FWHM }^{\mathrm{c}} \\
\left(\mathrm{km} \mathrm{s}^{-1}\right)\end{array}$ & $\begin{array}{c}\left.\log L_{[\mathrm{C}} \mathrm{II}\right] \\
\left(L_{\odot}\right)\end{array}$ & $\begin{array}{c}F_{[\mathrm{N} \mathrm{II}]} 205 \\
\left(10^{-18} \mathrm{~W} \mathrm{~m}^{-2}\right)\end{array}$ & $\begin{array}{c}\log L_{\left[\mathrm{N}_{\mathrm{II}} 205\right.} \\
\left(L_{\odot}\right)\end{array}$ \\
\hline BOOTES1 & $<6.6$ & $<8.85$ & $7.6 \pm 2.4$ & 305 & $8.91 \pm 0.13$ & $<7.2$ & $<8.89$ \\
\hline BOOTES2 & $\cdots$ & $\cdots$ & $12.5 \pm 3.5$ & $661 \pm 149$ & $8.61 \pm 0.52$ & $<4.8$ & $<8.42$ \\
\hline BOOTES3 & $\ldots$ & $\ldots$ & $47.7 \pm 5.6$ & $545 \pm 43$ & $9.22 \pm 0.04$ & $<6.0$ & $<8.32$ \\
\hline CDFS1 & $\cdots$ & $\cdots$ & $16.7 \pm 2.9$ & $764 \pm 132$ & $9.41 \pm 0.06$ & $<4.8$ & $<8.57$ \\
\hline CDFS2 & $\cdots$ & $\cdots$ & $20.5 \pm 2.8$ & $390 \pm 54$ & $9.04 \pm 0.05$ & $<6.0$ & $<8.46$ \\
\hline ELAISS & $\ldots$ & $\ldots$ & $22.1 \pm 2.1$ & $433 \pm 41$ & $9.13 \pm 0.03$ & $<4.8$ & $<8.43$ \\
\hline FLS01 & $<7.8$ & $<9.1$ & $3.8 \pm 1.10$ & 317 & $8.78 \pm 0.12$ & $<5.4$ & $<8.94$ \\
\hline FLS02 & $<5.7$ & $<9.0$ & $4.7 \pm 1.10$ & 317 & $8.92 \pm 0.10$ & $<4.8$ & $<8.93$ \\
\hline SWIRE1 & $<0.15$ & $<8.1$ & $<7.2$ & $\ldots$ & $<9.86$ & $<0.06$ & $<7.78$ \\
\hline SWIRE2 & $<6.3$ & $<8.6$ & $<9.3$ & $\ldots$ & $<9.03$ & $<5.1$ & $<8.77$ \\
\hline SWIRE4 & $\ldots$ & $\ldots$ & $24.2 \pm 3.5$ & $556 \pm 75$ & $9.04 \pm 0.04$ & $<1.5$ & $<7.86$ \\
\hline SWIRE5 & $4.04 \pm 0.12$ & $8.6 \pm 0.01$ & $27.8 \pm 2.1$ & $417 \pm 43$ & $9.41 \pm 0.03$ & $<4.5$ & $<8.72$ \\
\hline HLSW-01 (SWIRE6) & $<10.8 \mu$ & $<11.3$ & $(8.5 \pm 1.5) / \mu$ & 896 & $11.21 \pm 0.07$ & $\cdots$ & $\cdots$ \\
\hline XMM1 & $<11.1$ & $<11.0$ & $5.4 \pm 1.8$ & 740 & $10.76 \pm 0.14$ & $\cdots$ & $\cdots$ \\
\hline ELAISN $^{d}$ & $\ldots$ & $\ldots$ & $<5.40$ & $\ldots$ & $<9.47$ & $\ldots$ & $\ldots$ \\
\hline SWIRE7 $^{\mathrm{d}}$ & $<11.1$ & $<9.54$ & $<5.40$ & $\ldots$ & $<9.22$ & $<5.4$ & $<9.22$ \\
\hline $\mathrm{XMM} 2^{\mathrm{d}}$ & 3.2 & 8.78 & $<3.9$ & $\cdots$ & $<8.39$ & $<6.0$ & $<8.58$ \\
\hline
\end{tabular}

Notes.

${ }^{\mathrm{a}}$ In the cases of non-detections, we report $3 \sigma$ upper limits. No value indicates that the line is out of the FTS bandwidth for the redshift of the source.

${ }^{\mathrm{b}}$ In the cases of non-detection, we report $3 \sigma$ upper limits.

${ }^{\mathrm{c}}$ Uncertainties are quoted for sources where a gauss+sinc profile provides the best fit to the data. For the rest of the sources, we quote the FWHM that corresponds to the fixed FWHM $=1.4305 \mathrm{GHz}$ of the sinc function.

${ }^{\mathrm{d}}$ Sources with photometric redshift. The upper limits correspond to measurements at the expected frequency of the lines based on the photo- $z$ estimate.

Table 4

CO IRAM and APEX Observations

\begin{tabular}{|c|c|c|c|c|c|c|c|}
\hline$\overline{\text { Source }}$ & $z_{\mathrm{CO}}{ }^{\mathrm{a}}$ & Line & $\begin{array}{c}S_{\mathrm{CO}} \Delta v \\
\left(\mathrm{~J} \mathrm{~km} \mathrm{~s}^{-1}\right)\end{array}$ & $\begin{array}{l}\Delta V_{\mathrm{FWHM}} \\
\left(\mathrm{km} \mathrm{s}^{-1}\right)\end{array}$ & $\begin{array}{c}\log L_{\mathrm{CO}}^{\prime} \\
\left(\mathrm{K} \mathrm{km} \mathrm{s}^{-1} \mathrm{pc}^{2}\right)\end{array}$ & $\begin{array}{c}\log L_{\mathrm{IR}} \\
\left(L_{\odot}\right)\end{array}$ & $\begin{array}{c}\log L_{\mathrm{CO}[1-0]}^{\prime}{ }^{\mathrm{b}} \\
\left(\mathrm{K} \mathrm{km} \mathrm{s}^{-1} \mathrm{pc}^{2}\right)\end{array}$ \\
\hline \multirow[t]{2}{*}{ BOOTES1 } & 0.352 & $\mathrm{CO}[1-0]$ & $1.63 \pm 0.28$ & $184 \pm 43$ & $10.21 \pm 0.08$ & 12.69 & $10.21 \pm 0.08$ \\
\hline & 0.352 & $\mathrm{CO}[2-1]$ & $7.58 \pm 1.10$ & $416 \pm 71$ & $10.09 \pm 0.06$ & 12.69 & $10.21 \pm 0.08$ \\
\hline BOOTES2 & 0.249 & $\mathrm{CO}[1-0]$ & $2.01 \pm 0.38$ & $378 \pm 74$ & $9.81 \pm 0.08$ & 11.80 & $9.81 \pm 0.08$ \\
\hline BOOTES3 & 0.216 & $\mathrm{CO}[1-0]$ & $6.19 \pm 0.81$ & $627 \pm 82$ & $10.17 \pm 0.05$ & 11.87 & $10.17 \pm 0.05$ \\
\hline SWIRE4 & 0.248 & $\mathrm{CO}[1-0]$ & $3.40 \pm 0.57$ & $556 \pm 105$ & $10.04 \pm 0.07$ & 11.79 & $10.04 \pm 0.07$ \\
\hline \multirow[t]{2}{*}{ SWIRE5 } & 0.366 & $\mathrm{CO}[1-0]$ & $2.78 \pm 0.52$ & $476 \pm 145$ & $10.29 \pm 0.08$ & 12.06 & $10.29 \pm 0.08$ \\
\hline & 0.367 & $\mathrm{CO}[2-1]$ & $8.20 \pm 1.15$ & $531 \pm 104$ & $10.16 \pm 0.06$ & $"$ & " \\
\hline \multirow[t]{2}{*}{ FLS02 } & 0.436 & $\mathrm{CO}[2-1]$ & $3.36 \pm 0.81$ & $347 \pm 124$ & $9.93 \pm 0.04$ & 12.41 & $10.05 \pm 0.05$ \\
\hline & 0.436 & $\mathrm{CO}[3-2]$ & $5.76 \pm 1.10$ & $380 \pm 77$ & $9.81 \pm 0.08$ & " & $10.11 \pm 0.16$ \\
\hline CDFS1 & 0.289 & $\mathrm{CO}[3-2]$ & $11.15 \pm 2.29$ & $290 \pm 64$ & $9.72 \pm 0.08$ & 11.79 & $10.03 \pm 0.16$ \\
\hline CDFS2 & 0.248 & $\mathrm{CO}[3-2]$ & $17.05 \pm 5.57$ & $423 \pm 198$ & $9.77 \pm 0.14$ & 11.82 & $10.07 \pm 0.28$ \\
\hline ELAISS & 0.265 & $\mathrm{CO}[3-2]$ & $12.13 \pm 3.60$ & $273 \pm 150$ & $9.68 \pm 0.12$ & 11.59 & $9.98 \pm 0.24$ \\
\hline
\end{tabular}

Notes.

${ }^{\text {a }}$ Typical uncertainty, $\Delta z=0.001$.

${ }^{\mathrm{b}}$ For sources where $\mathrm{CO}[1-0]$ observations are not available, we adopt $r_{21}=0.75$ and $r_{32}=0.5$.

cases there is evidence of a double-horned profile. Considering a line detected when the integrated signal is larger than $>3 \sigma$, we have solid detections for at least one line per source. The median line width of our sample is $416 \mathrm{~km} \mathrm{~s}^{-1}$ and ranges from 238 to $538 \mathrm{~km} \mathrm{~s}^{-1}$. Given the angular distance of the sources (average value $1000 \mathrm{Mpc}$ ), our beam subtends between 50 and $100 \mathrm{kpc}$, and all galaxies can be considered unresolved, at least as far as their molecular component is concerned. All spectra along with the best-fit models are presented in Figure 3, while the properties of the fitted lines are summarized in Table 4.

Line luminosities, $L_{\mathrm{CO}}$ (measured in $L_{\odot}$ ), and $L_{\mathrm{CO}}^{\prime}$ (measured in $\mathrm{K} \mathrm{km} \mathrm{s}^{-1} \mathrm{pc}^{2}$ ) were derived using Equations (1) and (3) of Solomon \& Vanden Bout (2005)

$$
L_{\mathrm{CO}}=1.04 \times 10^{-3} S_{\mathrm{CO}} \Delta u v_{\mathrm{rest}}(1+z)^{-1} D_{\mathrm{L}}^{2},
$$

where $L_{\mathrm{CO}}$ is measured in $L_{\odot}$, the luminosity distance $D_{\mathrm{L}}$ is in megaparsecs, the velocity integrated flux, $S_{\mathrm{CO}} \Delta \mathrm{u}$, in $\mathrm{Jy} \mathrm{km} \mathrm{s}^{-1}$, the rest-frame frequency of the observed line, $v_{\text {rest }}$, in GHz, and

$$
L_{\mathrm{CO}}^{\prime}=3.25 \times 10^{7} S_{\mathrm{CO}} \Delta u v_{\mathrm{obs}}^{-2} D_{\mathrm{L}}^{2}(1+z)^{-3} .
$$

As discussed, for four sources we targeted and detected the CO $[1-0]$ emission line so the derived $L_{\mathrm{CO}}^{\prime}$ corresponds to $L_{\mathrm{CO}[1-01}^{\prime}$. For the remaining five sources (FLS2, BOOTES2, CDFS1, CDFS2, and ELAISS), we converted the observed $L_{\mathrm{CO}}^{\prime}=L_{\mathrm{CO}[2-1]}^{\prime}$ or $L_{\mathrm{CO}}^{\prime}=L_{\mathrm{CO}[3-2]}^{\prime}$ to $L_{\mathrm{CO}[1-0]}^{\prime}$, adopting $r_{21}=$ 0.75 and $r_{32}=0.5$ (e.g., Dannerbauer et al. 2009; Ivison et al. 2010a; Rigopoulou et al. 2013; Aravena et al. 2014). For XMM01 and HLSW-01 (SWIRE6), we adopt the CO[1-0] measurements reported by Fu et al. (2013) and Scott et al. (2011), respectively. 

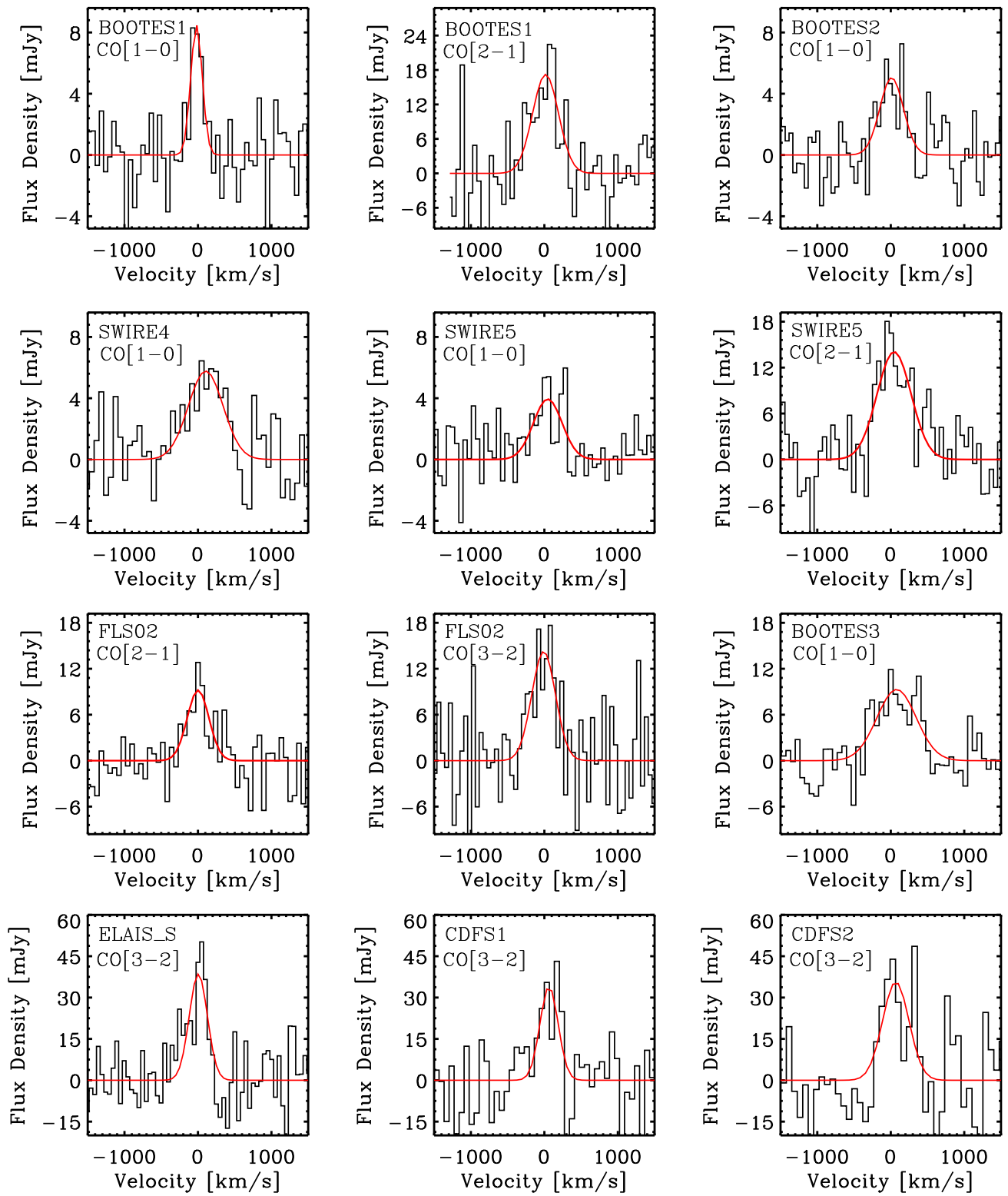

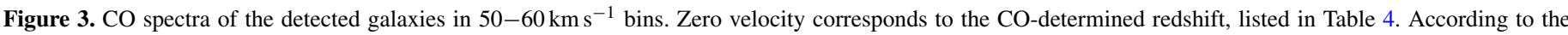

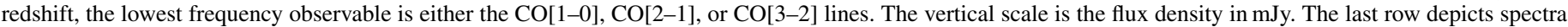
obtained by APEX, while the rest were obtained by IRAM.

(A color version of this figure is available in the online journal.)

\section{DERIVATION OF FAR-IR PROPERTIES}

We combine ancillary Spitzer IRAC $(3.6-8.0 \mu \mathrm{m})$ and MIPS $(24,70,160 \mu \mathrm{m})$ photometry with new Herschel SPIRE observations at 250,350, and $500 \mu \mathrm{m}$, and use the Draine \& Li (2007, hereafter DL07) model to derive dust masses and infrared luminosities by fitting the mid-IR to submillimeter photometry. The analysis is also supplemented by a more simplistic but widely used single temperature modified blackbody (MBB) fit in order to derive a representative single dust temperature $\left(T_{\mathrm{d}}\right)$ of the ISM. In particular, we adopt a fixed effective dust emissivity index of $\beta=1.5$ and fit observed data points with $\lambda_{\text {rest }}>50 \mu \mathrm{m}$ to avoid emission from very small grains that dominate at shorter wavelengths. A similar technique for the derivation of $M_{\text {dust }}, T_{\mathrm{d}}$, and $L_{\mathrm{IR}}$ using the DL07 and MBB models is presented in detail in Magdis et al. (2012a, 2013). The best-fit model SEDs are presented in Figure 4 and the corresponding derived parameters are summarized in Table 5.
The infrared luminosities $\left(L_{\mathrm{IR}}=L[8-1000 \mu \mathrm{m}]\right)$ of the galaxies in the sample (excluding the two high- $z$ lensed) vary between $3.8 \times 10^{11} L_{\odot}$ and $8.3 \times 10^{12} L_{\odot}$, with a median of $7.4 \times 10^{11} L_{\odot}$. Out of the entire $z<1$ spectroscopic sample, eight sources have $1.0 \times 10^{11}<L_{\mathrm{IR}} / L_{\odot}<1.0 \times 10^{12}$ and four sources $L_{\mathrm{IR}} / L_{\odot}>1.0 \times 10^{12}$. Similarly, we probe a wide range of dust temperatures, $T_{\mathrm{d}}=29-42 \mathrm{~K}$, and dust masses $M_{\text {dust }}=1.9 \times 10^{8}-2.8 \times 10^{9} M_{\odot}$.

However, we note that by imposing a flux cut limit in the $250 \mu \mathrm{m}$ flux density we are actually selecting sources based on their cold dust emission (for the redshift range of our targets), which could introduce a selection bias toward colder sources. This is depicted in Figure 5, where we show tracks of constant luminosity as a function of $T_{\mathrm{d}}$ and $S_{250}$ for galaxies at $z=$ 0.3 using an MBB with fixed $\beta=1.5$. At this redshift, our criterion selects sources with $L_{\mathrm{IR}} \leqslant 1.0 \times 10^{12} L_{\odot}$ and $T_{\mathrm{d}} \leqslant$ $40 \mathrm{~K}$, while missing warmer sources of similar $L_{\mathrm{IR}}$. On the other hand, at higher luminosities $\left(L_{\mathrm{IR}} \geqslant 2.5 \times 10^{12} L_{\odot}\right)$ we only miss 

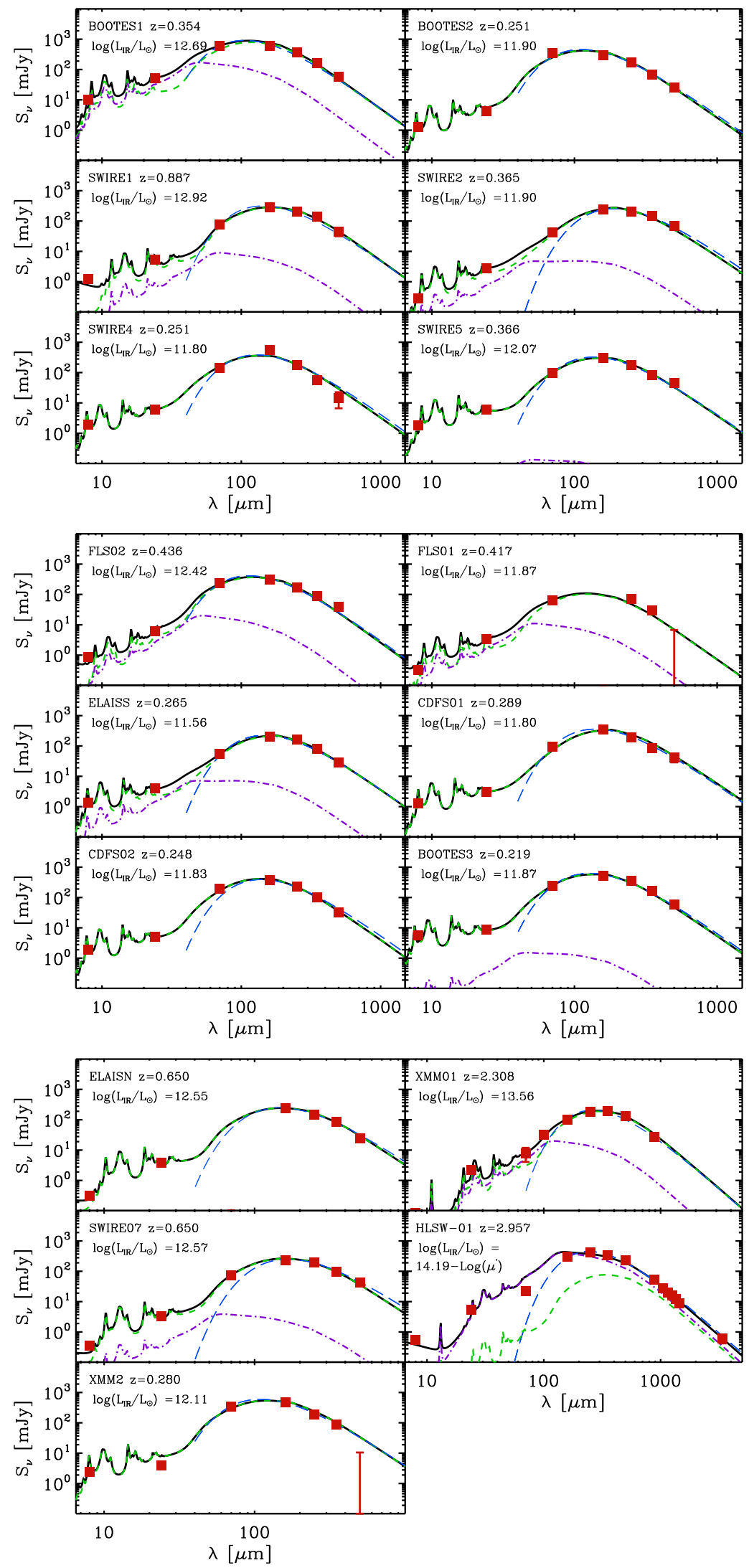

Figure 4. Spectral energy distributions (observed frame) of sources from our sample, including IRAC $8 \mu \mathrm{m}$, MIPS 24,70 , and $160 \mu \mathrm{m}$ (where available) and SPIRE 250,350 , and $500 \mu \mathrm{m}$ observed data points (red squares). The observed data are overlaid with the best-fit DL07 model (black line). The "PDR" and diffuse ISM components are shown in purple and green, respectively. The dashed blue line is the best-fit modified blackbody model with $\beta=1.5$. For XMM01 and HLSW-01, we also include (sub)millimeter photometry from Fu et al. (2013) and Conley et al. (2011), respectively.

(A color version of this figure is available in the online journal.) 
Table 5

Far-IR Properties

\begin{tabular}{lcccr}
\hline \hline Source & $\begin{array}{c}\log L_{\mathrm{IR}}{ }^{\mathrm{a}} \\
\left(L_{\odot}\right)\end{array}$ & $\begin{array}{c}\log L_{\mathrm{FIR}}{ }^{\mathrm{b}} \\
\left(L_{\odot}\right)\end{array}$ & $\begin{array}{c}\log M_{\mathrm{d}}{ }^{\mathrm{c}} \\
\left.M_{\odot}\right)\end{array}$ & $\begin{array}{c}T_{\mathrm{d}}{ }^{\mathrm{d}} \\
(\mathrm{K})\end{array}$ \\
\hline BOOTES1 & $12.69 \pm 0.02$ & $12.43 \pm 0.04$ & $9.06 \pm 0.16$ & $42 \pm 2$ \\
BOOTES2 & $11.91 \pm 0.05$ & $11.75 \pm 0.07$ & $8.61 \pm 0.16$ & $36 \pm 2$ \\
BOOTES3 & $11.87 \pm 0.04$ & $11.66 \pm 0.08$ & $8.92 \pm 0.14$ & $33 \pm 2$ \\
CDFS1 & $11.79 \pm 0.04$ & $11.55 \pm 0.07$ & $9.21 \pm 0.11$ & $29 \pm 2$ \\
CDFS2 & $11.82 \pm 0.03$ & $11.63 \pm 0.04$ & $8.87 \pm 0.07$ & $32 \pm 2$ \\
ELAISS & $11.59 \pm 0.04$ & $11.35 \pm 0.05$ & $8.87 \pm 0.14$ & $30 \pm 2$ \\
FLS01 & $11.86 \pm 0.03$ & $11.66 \pm 0.05$ & $8.29 \pm 0.14$ & $39 \mathrm{e}$ \\
FLS02 & $12.41 \pm 0.01$ & $12.24 \pm 0.03$ & $8.85 \pm 0.08$ & $42 \pm 2$ \\
SWIRE1 & $12.92 \pm 0.04$ & $12.74 \pm 0.07$ & $9.39 \pm 0.16$ & $39 \pm 2$ \\
SWIRE2 & $11.90 \pm 0.01$ & $11.62 \pm 0.02$ & $9.46 \pm 0.09$ & $28 \pm 2$ \\
SWIRE4 & $11.80 \pm 0.01$ & $11.60 \pm 0.02$ & $8.77 \pm 0.07$ & $33 \pm 3$ \\
SWIRE5 & $12.06 \pm 0.03$ & $11.85 \pm 0.06$ & $9.07 \pm 0.10$ & $33 \pm 2$ \\
HLSW-01 ${ }^{\mathrm{f}}(\mathrm{SWIRE6)}$ & $13.15 \pm 0.03$ & $12.98 \pm 0.07$ & $9.01 \pm 0.13$ & $51 \pm 2$ \\
XMM1 & $13.56 \pm 0.03$ & $13.36 \pm 0.04$ & $10.07 \pm 0.23$ & $39 \pm 2$ \\
\hline ELAISN & $12.54 \pm 0.05$ & $12.37 \pm 0.08$ & $9.15 \pm 0.22$ & $37 \pm 2$ \\
SWIRE7 & $12.56 \pm 0.05$ & $12.38 \pm 0.08$ & $9.27 \pm 0.18$ & $36 \pm 2$ \\
XMM2 & $12.11 \pm 0.05$ & $11.94 \pm 0.09$ & $8.83 \pm 0.21$ & $38 \pm 2$ \\
& & & &
\end{tabular}

Notes.

${ }^{\mathrm{a}} L_{\mathrm{IR}}=L[8-1000 \mu \mathrm{m}]$.

${ }^{\mathrm{b}} L_{\mathrm{FIR}}=L[42.5-122.5 \mu \mathrm{m}]$.

${ }^{c}$ Dust masses as derived from Draine \& Li (2007) models.

${ }^{\mathrm{d}}$ Dust temperatures as derived from modified blackbody fits with fixed $\beta=1.5$

for sources with at least three photometric data points at $\lambda_{\text {rest }}>50 \mu \mathrm{m}$.

e Based on the $L_{60} / L_{100}$ ratio from the best-fit DL07 model.

${ }^{\mathrm{f}}$ Values corrected for magnification assuming $\mu=10.9$ (Gavazzi et al. 2011)

extremely warm sources $\left(T_{\mathrm{d}} \geqslant 60 \mathrm{~K}\right)$. To compare our selection to samples in the pre-Herschel era, we repeat the analysis, this time considering the IRAS $60 \mu \mathrm{m}$ flux density. The blue shaded area in Figure 5 shows the parameter space covered by IRAS observations at a detection limit of $0.4 \mathrm{Jy}$ at $60 \mu \mathrm{m}$ (Faint Source Catalog; Moshir et al. 1992). Our flux cut threshold would select the majority of the IRAS-detected ULIRGs only missing warm sources $\left(T_{\mathrm{d}}>40 \mathrm{~K}\right)$ in the narrow luminosity range of $1-2.5 \times$ $10^{12} L_{\odot}$. However, given the sensitivity limitations of IRAS, at this redshift range the IRAS samples were predominantly limited to sources with $L_{\mathrm{IR}}>3 \times 10^{12} L_{\odot}$. As an example, all $0.2<z<0.6$ IRAS-selected ULIRGs presented by Combes et al. (2011) with $12.44<\log \left(L_{\mathrm{IR}} / L_{\odot}\right)<13.28$ and $36.4 \mathrm{~K}<T_{\mathrm{d}}<$ $56.6 \mathrm{~K}$ would meet out our $S_{250}$ flux cut limit. ${ }^{27}$ Furthermore, Symeonidis et al. (2013), using both IRAS- and Herschelselected samples, report that the majority of (U)LIRGs $\left(L_{\mathrm{IR}}>\right.$ $\left.10^{11} L_{\odot}\right)$ at all redshifts have mean dust temperatures between 25 and $45 \mathrm{~K}$, with $T_{\mathrm{d}}<25 \mathrm{~K}$ and $T_{\mathrm{d}}>45 \mathrm{~K}$ sources being rare. As stated above, the $T_{\mathrm{d}}$ range of our sample is $29-42 \mathrm{~K}$, very similar to the range of dust temperatures found by Symeonidis et al. (2013) for the bulk of the sources with infrared bolometric output comparable to that of the sources in our sample. We therefore conclude that our selection should be regarded fairly representative of the whole (U)LIRG population at the $0.2<z<$ 0.8 redshift range.

\section{THE PROPERTIES OF THE ISM OF INTERMEDIATE REDSHIFT (U)LIRGS}

\subsection{The $L_{C \mathrm{II}}-L_{I R}$ Relation Over Cosmic Time}

Various Galactic and extragalactic studies of the [C II] emission (e.g., Tielens \& Hollenbach 1985; Stacey et al. 1991;

\footnotetext{
27 None of these sources were selected in our sample as they were not covered by the Herschel HerMES observations.
}

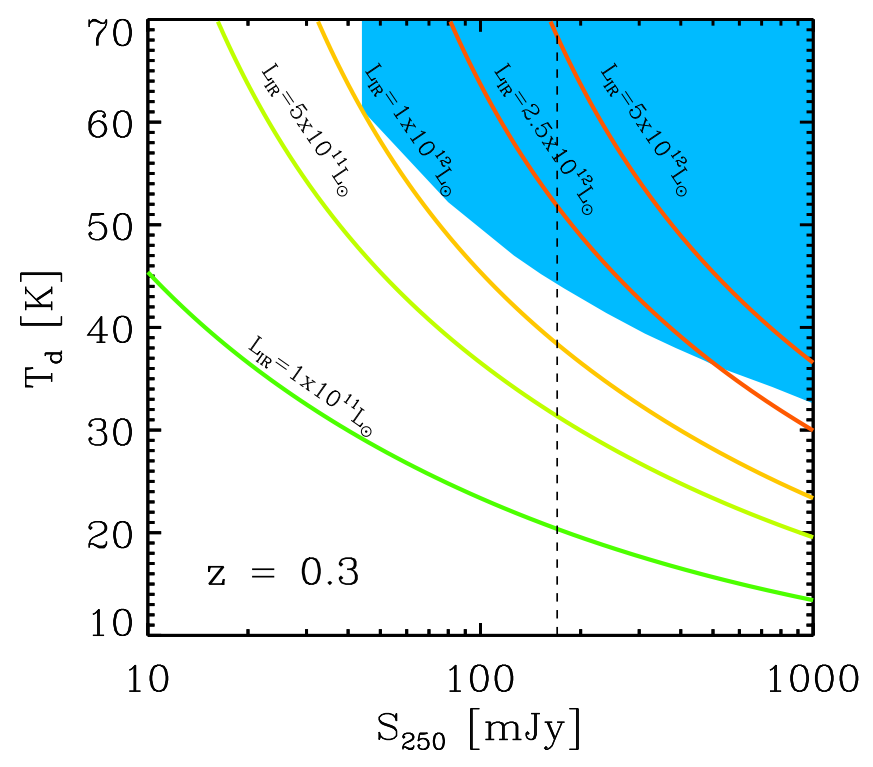

Figure 5. Dust temperature as a function of observed SPIRE $250 \mu$ m flux density as derived from a modified blackbody model with fixed $\beta=1.5$ of a source at $z=0.3$. The different colors correspond to tracks of constant $L_{\mathrm{IR}}$, ranging from $L_{\mathrm{IR}}=1 \times 10^{11} L_{\odot}$ (green line) to $L_{\mathrm{IR}}=5 \times 10^{12} L_{\odot}$ (red line). The vertical line corresponds to $S_{250}=170 \mathrm{mJy}$, which is the selection criterion for our sample. The blue shaded region depicts the parameter space obtained from IRAS observations at a detection threshold of $S_{60}=0.4 \mathrm{Jy}$.

(A color version of this figure is available in the online journal.)

Meijerink et al. 2007; Rigopoulou et al. 2013) have established that it predominantly originates from photodissociation regions (PDRs) in the outer layers of molecular clouds exposed to intense FUV radiation and have put it forward as a potentially powerful tracer of the star formation activity. Indeed, using $L_{\mathrm{FIR}}\left(L_{\mathrm{FIR}}=L_{42.5-122.5 \mu \mathrm{m}}\right)$ as an SFR indicator (e.g., Kennicutt et al. 1998), the majority of normal galaxies in the local universe seem to obey a universal linear $L_{\mathrm{CII}}-L_{\mathrm{FIR}}$ relation with a constant $L_{\mathrm{CII}} / L_{\mathrm{FIR}}$ ratio, albeit with a significant scatter (e.g., Malhotra et al. 2001). However, extending the study of [C $\mathrm{II}]$ in local (U)LIRGs has revealed that they appear to deviate from this relation exhibiting lower $L_{\mathrm{CI}} / L_{\mathrm{FIR}}$ ratios, i.e., they are [C II] deficient with respect to their infrared luminosity (e.g., Malhotra et al. 1997; Luhman et al. 1998, 2003; Diaz-Santos et al. 2013; Farrah et al. 2013).

Extrapolating observations of the local universe to high- $z$ galaxies, a natural expectation would be that high- $z$ (U)LIRGs would also appear [ $\left.\mathrm{C}_{\mathrm{II}}\right]$ deficient with respect to local normal galaxies. However, the detections of [C II] emission from distant $(z>1)$, star-forming galaxies have come to challenge this picture, revealing that ULIRGs can exhibit $L_{\mathrm{C} \text { II }} / L_{\mathrm{FIR}}$ ratios similar to that of local normal galaxies and a factor of $\sim 10$ larger compared to local (U)LIRGs (e.g., Hailey-Dunsheath et al. 2010; Stacey et al. 2010; Valtchanov et al. 2011; Riechers et al. 2013). While these studies seem to suggest a strong evolution in the [C II] emission between local $(z<0.2)$ and $z>1$ (U)LIRGs, it is unclear what drove this evolution.

Rigopoulou et al. (2014) found that the majority of Herschel-selected (U)LIRGs have $L_{\mathrm{CII}} / L_{\mathrm{FIR}}$ ratios similar to that of high- $z$ star-formation-dominated ULIRGs and local normal galaxies, suggesting that the transition of the properties in the ISM of the (U)LIRG phenomenon was already in place by $z \sim 0.3$. Here, by using the same sample, we bridge the gap between the local and high- $z$ galaxies and provide a continuous sampling of the $L_{\mathrm{C} \text { II }}-L_{\mathrm{FIR}}$ relation over the last 10 billion years for a wide range of extragalactic sources. 


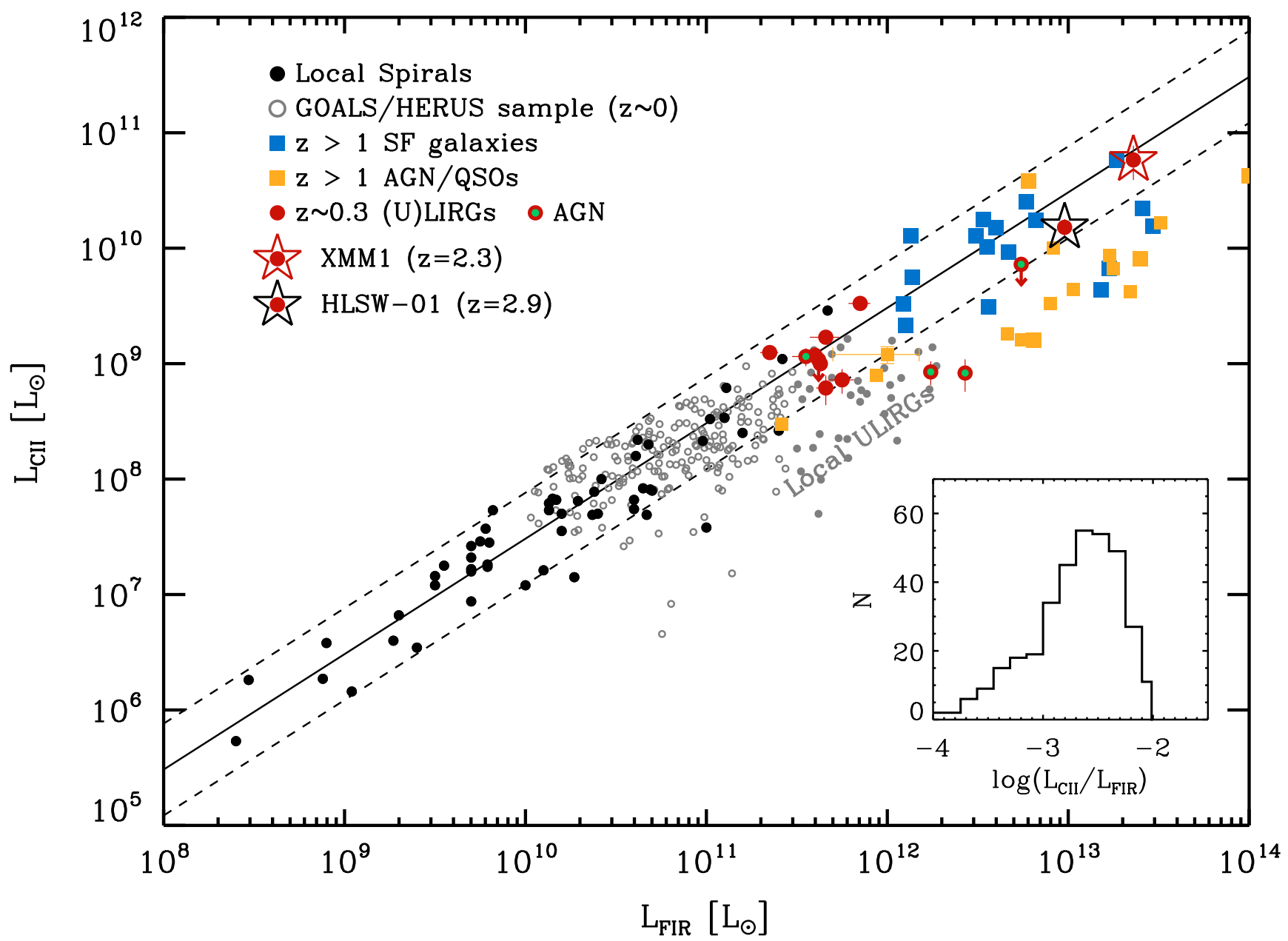

Figure 6. [C II] emission line luminosity $\left(L_{\mathrm{CII}}\right)$ vs. far infrared $(42.5-122.5 \mu \mathrm{m})$ luminosity $\left(L_{\mathrm{FIR}}\right)$. Our sample of intermediate redshift (U)LIRGs is shown as red circles. Sources from our sample with an AGN are indicated with a green filled circle, undetected sources are downward arrows ( $3 \sigma$ upper limits), and XMM01 and HLSW-01 as large stars. The rest of the data are local normal galaxies (filled black circles, from Malhotra et al. 2001), local (U)LIRGs from the GOALS and HERUSS samples (empty gray circles, from diaz-Santos et al. 2013 and Farrah et al. 2013), high- $z(z>1)$ star-forming galaxies (blue squares, from Stacey et al. 2010, Hailey-Dunsheath et al. 2010, Valtchanov et al. 2011, Ivison et al 2010a, Cox et al. 2011, De Breuck et al. 2011, Swinbank et al. 2012, George et al. 2013, Riechers et al. 2013, Rawle et al. 2014, Ferkinhoff et al. 2014, and Riechers et al. 2014), and high-z, AGN-dominated sources and QSOs (filled orange squares, from Iono et al. 2006, Walter et al. 2009, Wagg et al. 2010, Gallerani et al. 2012, Wang et al. 2012, Venemans et al. 2012, Carilli et al. 2013, and Willott et al. 2013). Gray filled circles correspond to local (U)LIRGs with $L_{I R}>5 \times 10^{11} L_{\odot}$. The solid black lines correspond to the best linear fit to the local normal galaxies of Malhotra et al. (2001) with a scatter of 0.3 dex (dashed lines) and a slope of unity. The inset panel shows the distribution of the $L_{\mathrm{C} \text { II }} / L_{\mathrm{FIR}}$ ratio for the all sources included in this plot.

(A color version of this figure is available in the online journal.)

In Figure 6, we plot the [C II] line luminosity as a function of far-infrared luminosity for local normal galaxies (Malhotra et al. 2001), local (U)LIRGs from the GOALS and HERUS samples (Diaz-Santos et al. 2013; Farrah et al. 2013), high- $z(z>1)$ starforming galaxies (Stacey et al. 2010; Hailey-Dunsheath et al. 2010; Valtchanov et al. 2011; Cox et al. 2011; Ivison et al 2010a; Swinbank et al. 2012; Rawle et al. 2013; George et al. 2013; Riechers et al. 2013; Ferkinhoff et al. 2014), high-z AGNs and QSOs (Iono et al. 2006; Wagg et al. 2010; Gallerani et al. 2012), and for our sample of $z \sim 0.3$ (ULIRGs). Fitting the local normal galaxies yields

$$
\log \left(L_{\mathrm{CII}}\right)=(-2.51 \pm 0.31)+\log \left(L_{\mathrm{FIR}}\right)
$$

revealing a constant $L_{\mathrm{CI}} / L_{\mathrm{FIR}}$ ratio albeit with a considerable scatter. As shown in Figure 6, the majority of star-forming galaxies (with $[\mathrm{C} \mathrm{II}]$ measurements) at all redshifts and infrared luminosities follow this relation (within the scatter), suggesting that $L_{\mathrm{C} \text { II }}$ can trace $L_{\mathrm{FIR}}$ and therefore the SFR within a factor of 2.5 at all epochs. However, as discussed above and as evident from the skewed tail of the $L_{\mathrm{C} \text { II }} / L_{\mathrm{FIR}}$ distribution (see the inset panel of Figure 6), outliers from this relation do exist and are predominantly the local (U)LIRGs $\left(L_{\mathrm{FIR}}>5 \times 10^{11} L_{\odot}\right)$, high$z$, AGN-dominated sources, and a small fraction of high- $z$ starforming galaxies. These sources appear [C II] deficient with respect to their $L_{\mathrm{FIR}}$ exhibiting, on average, a lower $L_{\mathrm{C} \text { II }} / L_{\mathrm{FIR}}$ ratio compared to that of local normal galaxies and the majority of high- $z$ star-formation-dominated ULIRGs. This trend implies an evolution in the star-forming regions of (U)LIRGs. Indeed, focusing on our sample of intermediate redshift (U)LIRGs, we find that only 2 out of the 14 targets appear to be [C II] deficient, while the rest exhibit $L_{\mathrm{C} \text { II }} / L_{\mathrm{FIR}}$ ratios similar to that of local normal galaxies and high- $z$ star-forming galaxies. Interestingly, these two sources are also classified as AGNs. In what follows, we will investigate the impact of an AGN on the observed $L_{\mathrm{C} \text { II }} / L_{\mathrm{FIR}}$ ratio of our sample.

\subsection{The Effect of an AGN}

A possible explanation for deviations from the general $L_{\mathrm{C} \text { II }}-L_{\mathrm{FIR}}$ trend could be the strong contribution of an AGN 


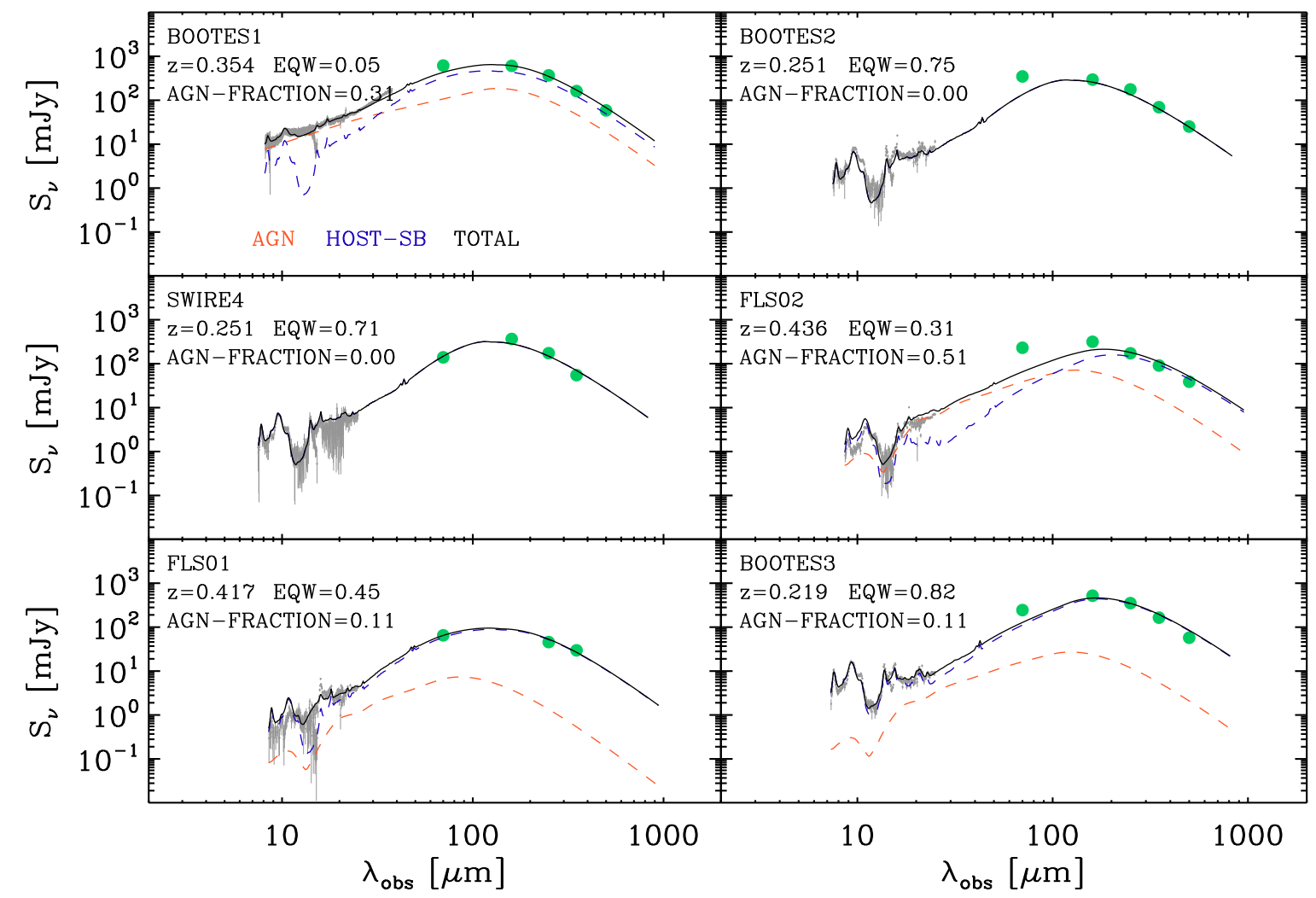

Figure 7. SED fitting using the AGN/host star-forming galaxy decomposition technique of Mullaney et al. (2011) for sources in our sample with available mid-IR IRS spectroscopy (gray points). Green circles show the observed far-IR photometric points and are overlaid with the best-fit total model (black line). The AGN and host galaxy components are shown as red and blue dashed lines, respectively. The quoted EQW corresponds to the equivalent width of the $6.2 \mu \mathrm{m}$ PAH feature.

(A color version of this figure is available in the online journal.)

to the bolometric infrared output of the source. If $L_{\mathrm{C}_{\text {II }}}$ is predominantly associated with star formation, while $L_{\mathrm{FIR}}$ arises from a mixture of AGNs and star formation activity, it would result in a decreased $L_{\mathrm{CII}} / L_{\mathrm{FIR}}$ ratio with respect to purely starforming galaxies. This scenario holds under the assumption that even if [C II] can be excited by AGN activity (both in PDRs and $\mathrm{XDRs}$ ), its contribution to the total [C $\mathrm{CI}]$ emission is typically found to be small (e.g., Crawford et al. 1985; Unger et al. 2000; Stacey et al. 2010).

To investigate this scenario, we perform an AGN/host galaxy decomposition for five sources in our sample that benefit from mid-IR IRS spectra, using the SED decomposition method of Mullaney et al. (2011). This method employs a host-galaxy and an intrinsic AGN template SED to measure the contribution to the infrared output of these two components. The technique identifies the best-fitting model SED for the observed infrared data (spectra and photometry) through $\chi^{2}$ minimization and by varying the values of a set of free parameters (for details, see Magdis et al. 2013). The best-fit model SEDs are shown in Figure 7. Out of the five sources with available IRS spectra, two are classified as AGNs (Bootes1 and FLS2) and three as starforming or composites based on optical or mid-IR spectroscopy.

From the various output parameters, we focused on the contribution of an AGN in the far-infrared emission $\left(f_{\mathrm{AGN}}\right)$. For star-forming and composite sources (classified based on the $\mathrm{EW}$ of the $6.2 \mu \mathrm{m}$ PAH feature), we find a negligible (if any) contribution of an AGN to $L_{\mathrm{FIR}}$. For the two AGNs, which also happen to have $L_{\mathrm{C} \text { II }} / L_{\mathrm{FIR}}$ similar to that of local ULIRGs, we derive $f_{\mathrm{AGN}}=0.31$ (BOOTES1) and $f_{\mathrm{AGN}}=0.51$ (FLS2). We note that given the poor fit to the rest-frame mid-IR data for these two sources, these values should be treated with caution and the exact contribution of an AGN to the $L_{\mathrm{FIR}}$ remains largely unconstrained. However, even if we correct the $L_{\mathrm{FIR}}$ by a factor of two the $L_{\mathrm{C}} / L_{\mathrm{FIR}}$ of these two sources remain approximately $\sim 3$ times lower compared to the rest of the sample. Therefore, while the existence of an AGN can partially affect the $L_{\mathrm{C} \text { II }} / L_{\mathrm{FIR}}$ values for these systems, the AGN contribution to $L_{\mathrm{FIR}}$ cannot fully explain the observed [C II] deficit. Furthermore, the rest of the sources in our sample that are classified as AGNs fall within the scatter of the $L_{\mathrm{CII}}-L_{\mathrm{FIR}}$ relation defined in Equation (3), suggesting that there is no direct correlation between the existence of an AGN and the observed $L_{\mathrm{C}} / L_{\mathrm{FIR}}$ ratio. Similar results have been reached by Diaz-Santos et al. (2013) and Farrah et al. (2013) based on a sample of local ULIRGs and using a number of far-IR lines. However, we should stress that in our sample, as well as in the majority of local ULIRGs, the far-IR output is dominated by star formation (e.g., Genzel et al. 1998; Rigopoulou et al. 1999; Armus et al. 2007; Desai et al. 2007; Petric et al. 2011). On the other hand, sources where AGN activity dominates the far-IR output of the sources, both in the local universe as well as at high- $z$, the origin of the [C II] deficit can be explained through the contamination of $L_{\mathrm{FIR}}$ by AGN activity (e.g., Stacey et al. 2010; Wagg et al. 2010; Sargsyan et al. 2012).

Therefore, we cannot rule out that for sources where AGN activity dominates the far-IR output of the sources, both in the local universe as well as at high- $z$, the origin of the [C II] deficit as reported in various studies is not due to contamination of $L_{\mathrm{IR}}$ by AGN activity (e.g., Stacey et al. 2010; Wagg et al. 2010; Sargsyan et al. 2012). 

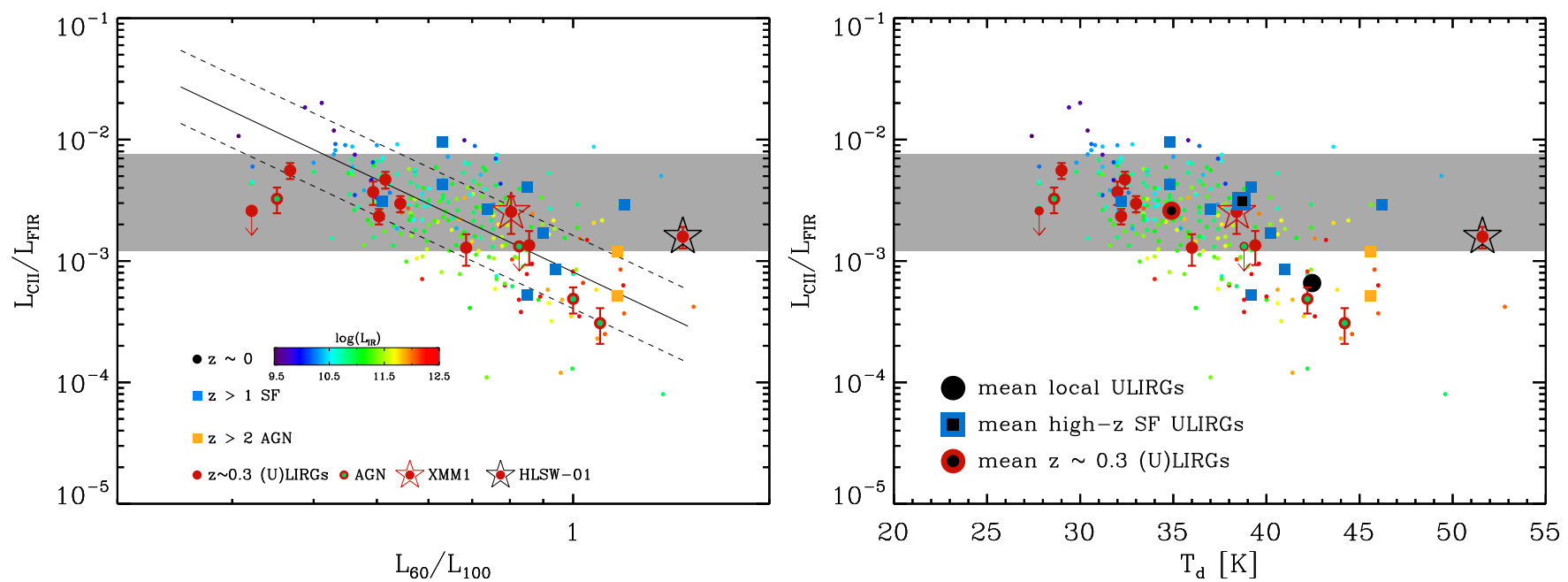

Figure 8. Left: ratio of [C $\mathrm{CI}]$ line luminosity to far-infrared luminosity ( $L_{\mathrm{FIR}}$ ) as a function of the $L_{60} / L_{100}$ continuum luminosity ratio for local (U)LIRGs (GOALS and HERUS), intermediate redshift (U)LIRGs, high- $z$ star-forming galaxies, and high- $z$ QSOs. The local sample is color-coded based on the luminosity of the sources. The plot reveals a decrease of the $L_{\mathrm{C} \text { II }} / L_{\mathrm{FIR}}$ ratio for warmer sources at all redshifts. The solid line corresponds to a linear fit of the all data in log-log space. The parameters of the fit are given in Equation (6). The dotted lines correspond to the $\pm 1 \sigma$ uncertainty of the derived relation. The vertical shaded region covers the range of $L_{\mathrm{C} \text { II }} / L_{\mathrm{FIR}}$ values within $1 \sigma$ of eq. (3). Right: same as the left panel, but using a modified blackbody with fixed $\beta=1.5$ and the redshift of the sources to convert the observed continuum luminosity ratio to dust temperature $\left(T_{\mathrm{d}}\right)$. We also include the mean values for the local, intermediate redshift, and high- $z$ ULIRGs.

(A color version of this figure is available in the online journal.)

\section{3. $L_{C \text { II }}-L_{F I R}$ as a Function of $T_{d}$}

Various studies of $[\mathrm{CII}]$ emission from normal galaxies and local (U)LIRGs have established an observational trend where sources with lower $L_{\mathrm{CII}} / L_{\mathrm{FIR}}$ values tend to have warmer dust temperatures (e.g., Malhotra et al. 1997). Since the $L_{\text {CII }} /$ $L_{\mathrm{FIR}}$ ratio depends on the efficiency of the heating of gas by photoelectrons from dust, it is indeed expected to be sensitive to the physical conditions of the ISM. A possible explanation for the observed trend was offered by Malhotra et al. (1997) based on the study of the colors and the [C II] emission of local ULIRGs. They suggested that the [C II] deficit of warmer sources could be explained by a high $G_{0} / n_{\mathrm{H}}$ ratio in the neutral galactic medium, where $G_{0}$ is the incident far-UV $6-13.6 \mathrm{eV}$ radiation field and $n_{\mathrm{H}}$ the total hydrogen gas density. In this scenario, more extreme $G_{0} / n_{\mathrm{H}}$ ratios would lead to an increase in the positive charge of the dust particles, which would result in a reduction of the amount of photo-electrons (with sufficient kinetic energy) released from dust grains, and consequently, in a decrease of the efficiency in the transformation of the incident UV radiation into gas heating.

In a more general scenario, the observed deficits of various far-IR atomic lines could be the result of a higher average ionization parameter of the ISM, $\langle U\rangle$ (Luhman et al. 2003; Abel et al. 2009), defined as the dimensionless ratio of the incident ionizing photon density to the hydrogen density

$$
U=\frac{Q_{\mathrm{H}}}{4 \pi R^{2} n_{\mathrm{H}} c},
$$

where $Q_{\mathrm{H}}$ is the number of hydrogen ionizing photons, $R$ is the distance of the ionizing sources to the PDR, and $c$ is the speed of light. Assuming an average stellar population and size of star-forming regions, the above relation yields

$$
U \propto \frac{G_{0}}{n_{\mathrm{H}}} .
$$

For fixed $n_{\mathrm{H}}$, higher levels of ionization (i.e., higher $\langle U\rangle$ values) would result in a higher ratio of ionized to atomic hydrogen and therefore in a decreased gas opacity in the H II regions, since less hydrogen atoms will be available to absorb UV photons. Subsequently, this will lead to a decreased gas-to-dust opacity, as dust will have to compete for UV photons with fewer neutral hydrogen atoms in the $\mathrm{H}$ II regions. As a consequence, a significant fraction of the UV radiation is eventually absorbed by large dust grains before being able to reach the neutral gas in the PDRs and ionize the PAH molecules (Voit 1992; Abel et al. 2009), causing a deficit of photo-electrons and hence, suppression of the [C II] line with respect to the total FIR dust emission. Higher $\langle U\rangle$ values will also translate into larger numbers of photons per dust particle and therefore higher dust temperature or warmer far-IR colors for sources with lower $L_{\mathrm{C} \text { II }} / L_{\mathrm{FIR}}$ (or other atomic lines) ratios. We note that the assumption of fixed $n_{\mathrm{H}}$ appears to be valid based on the observation that $\left(L_{\mathrm{OI}}+L_{\mathrm{CII}}\right) / L_{\mathrm{FIR}}$ varies as a function of far-IR color $\left(S_{60} / S_{100}\right)$.

In Figure 8, we plot the $L_{\mathrm{C} \text { II }} / L_{\mathrm{FIR}}$ ratio as a function of the $L_{60} / L_{100}$ color and dust temperature for the same sample of galaxies as in Figure 6. For the local galaxies we used the available IRAS photometry, while for our intermediate- $z$ and high- $z$ literature sources we convolved the best-fit SED with the IRAS filters. To convert the observed flux ratios of the sources drawn form the literature to dust temperatures, we assumed a MBB with a fixed dust emissivity index $\beta=1.5$. In both panels, the local sample is color-coded based on the infrared luminosity of the sources. It appears that the strong anti-correlation between the $L_{\mathrm{C} \text { II }} / L_{\mathrm{FIR}}$ ratio and the dust temperature, as already observed in the local universe by various authors (e.g., Diaz-Santos et al. 2013), holds at all luminosities and all redshifts. The best fit to the data yields the relation

$$
\log \left(\frac{L_{\mathrm{C} \text { II }}}{L_{\mathrm{FIR}}}\right)=(-3.09 \pm 0.06)+(-2.53 \pm 0.21) \log \left(\frac{L_{60}}{L_{100}}\right)
$$

with a scatter of 0.3 dex, suggesting a strong dependence between the $L_{\mathrm{C} \text { II }} / L_{\mathrm{FIR}}$ ratio and the dust temperature at all epochs, with warmer sources exhibiting lower $L_{\mathrm{C} \text { II }} / L_{\mathrm{FIR}}$ ratios. 
This trend also provides hints about the physical origin of the observed difference of the $L_{\mathrm{C} \text { II }} / L_{\mathrm{FIR}}$ ratio between the local and distant ULIRGs. Several studies have shown that the majority of high- $z$ star-forming galaxies with ULIRG-like luminosities are colder than galaxies in the local universe with similar $L_{\mathrm{IR}}$ (e.g., Hwang et al. 2010; Magdis et al. 2010, 2012b; Symeonidis et al. 2013). This is clearly depicted in Figure 8, where the bulk of high- $z$ star-forming galaxies from the literature that have [C II] measurements exhibit lower $L_{60} / L_{100}$ with respect to local ULIRGs. Note that all high- $z$ star-forming galaxies in this sample have $L_{\mathrm{IR}}>10^{12} L_{\odot}$. Similarly, we find that our sample of Herschel-selected intermediate redshift (U)LIRGs are on average colder than local ULIRGs with $\left\langle T_{\mathrm{d}}\right\rangle=34 \mathrm{~K}$ compared to $42 \mathrm{~K}$ for local ULIRGs.

As discussed above, the observed trend is compatible with the physical scenario in which the variation of $L_{\mathrm{CII}} / L_{\mathrm{FIR}}$ is caused by an increase of $\langle U\rangle$ in [C II] deficient sources. Diaz-Santos et al. (2013; Díaz-Santos et al. 2014), showed that for local (U)LIRGs the observed decrease of $L_{\text {CII }} / L_{\text {FIR }}$ ratio in warmer sources, i.e., sources with higher $\langle U\rangle$, is linked to the luminosity surface density of the mid-IR emitting region; warm, [C II] deficient sources are associated with compact starbursts. Furthermore, based on resolved [C II] observations, they found that $\left[\mathrm{C}_{\mathrm{II}}\right]$ deficient regions of local luminous LIRGs are restricted to their nuclei with extra nuclear emission showing $L_{\mathrm{C} \text { II }} / L_{\mathrm{FIR}}$ ratios similar to that of normal galaxies. The implications of these findings for the high- $z$ galaxies are discussed in Section 6.

Finally, we note that variation of the $L_{\mathrm{C} \text { II }} / L_{\mathrm{FIR}}$ ratio could also be driven by galactic scale metallicity effects. For example, higher dust temperatures could result from the increased hardness of the radiation field and the transparency of the ISM toward lower metallicities, which enhances the ionization of gas. In combination with the longer mean free path lengths of ionizing photons in metal-poor galaxies, the filling factors of ionized gas media are bound to grow drastically (e.g., De Looze et al. 2014). Furthermore, more UV photons might reach the neutral PDR gas in low metal abundance environments, where the hard radiation field reduces the $\mathrm{PAH}$ abundance and/or charges dust grains in PDRs, resulting in a reduced [C II] line emission. Apart from the CII deficit, metallicity variations could also be responsible for the observed scatter in the $L_{\mathrm{C} \text { II }}-L_{\mathrm{FIR}}$ relation.

\section{4. $L_{C}{ }_{\mathrm{II}}-L_{I R}$ and Star Formation Efficiency}

In a recent study, Graciá-Carpio et al. (2011) reported a strong anti-corellation between the relative strength of the farIR lines and the infrared luminosity to molecular gas mass ratio, $L_{\mathrm{IR}} / M_{\mathrm{H}_{2}}$. In particular, they argued that galaxies with high SFEs $\left(L_{\mathrm{IR}} / M_{\mathrm{H}_{2}}\right.$; Sanders \& Mirabel 1985) tend to have weaker farIR emission lines as a consequence of an increased ionization parameter. Indeed, in a simplified scenario, the radiation field should be proportional to the number of available ionizing photons $\left(L_{\mathrm{IR}}\right)$ per hydrogen molecule $\left(M_{\mathrm{H}_{2}}\right)$. They concluded that for fixed hydrogen density, the observed [C II] deficit that starts to appear at $L_{\mathrm{IR}} / M_{\mathrm{H}_{2}}>80 L_{\odot} / M_{\odot}$ can be understood if the ionization parameter is an order of magnitude larger compared to sources with lower $L_{\mathrm{IR}} / M_{\mathrm{H}_{2}}\left(\langle U\rangle \sim 10^{-3}\right.$ to $10^{-2.5}$ for the latter). Interestingly, the value of $L_{\mathrm{IR}} / M_{\mathrm{H}_{2}} \approx 80 L_{\odot} / M_{\odot}$ where the far-IR line deficits start to manifest is also similar to the limit that separates between the two modes of star formation (merger driven starbursts versus normal quiescent galaxies, e.g., Daddi et al. 2010a, 2010b; Genzel et al. 2010). Here, we test this scenario for our sample of intermediate redshift (U)LIRGs by using our $\mathrm{CO}$ and $M_{\text {dust }}$ measurements.

A great source of uncertainty in this investigation is the derivation of $M_{\mathrm{H}_{2}}$ estimates that are dependent on the adopted CO-to- $M_{\text {gas }}$ conversion factor $\left(\alpha_{\mathrm{CO}}\right)$, which is known to vary by a factor of six between local normal galaxies and local ULIRGs (e.g., Downes \& Solomon 1998; Bolatto et al. 2013). Here, instead of using a single $\alpha_{\mathrm{CO}}$ value for all sources, we take advantage of the dust mass estimates for our galaxies and employ the dust to gas mass ratio technique (e.g., Magdis et al. 2011a, 2012b). Adopting a solar metallicity and the dust to gas mass ratio versus metallicity relation $\left(\delta_{\mathrm{GDR}}-Z\right)$ of Leroy et al. (2011), we find an $M_{\text {gas }} / M_{\text {dust }}$ ratio of $\sim 80$. Then, using the $M_{\text {dust }}$ estimates for our sample, we derive the gas mass and the corresponding error for our sources, taking into account the $M_{\text {dust }}$ uncertainties, as well as assuming a 0.2 dex uncertainty in the adopted metallicity and therefore in the assumed $M_{\mathrm{gas}} /$ $M_{\text {dust }}$ ratio. A full description of the limitations inherent in this approach are presented in Magdis et al. (2012b).

In Figure 9 (left), we plot the $L_{\mathrm{C} \text { II }} / L_{\mathrm{FIR}}$ ratio as a function of $L_{\mathrm{IR}} / M_{\mathrm{H}_{2}},{ }^{28}$ focusing on the sample for which we have [C II] and $[\mathrm{CO}]$ measurements. This figure reveals a clear trend, with sources that deviate more than $1 \sigma$ from the $L_{\mathrm{C} \text { II }}-L_{\mathrm{FIR}}$ relation of Equation (3) exhibiting the highest SFEs. A Kendal's tau ranking test yields a correlation coefficient of $\rho=-0.9$ and a $p$-value of $4 \times 10^{-5}$, suggesting a statistically significant anticorrelation between the two parameters. For comparison, we also include a sample of local disks and local ULIRGs, adopting an $\alpha_{\mathrm{CO}}{ }^{29}$ conversation factor of 4.5 and 0.8 , respectively (e.g., Downes \& Solomon 1998; Rosolowsky et al. 2007; Bolatto et al. 2008, 2013; Sliwa et al. 2012). The majority of intermediate redshift (U)LIRGs in our sample fall in the locus of local disks, while the two [C II] deficient sources have SFEs similar to that of local ULIRGs. Similar to Graciá-Carpio et al. (2011), we conclude that low $L_{\mathrm{C} \text { II }} / L_{\mathrm{FIR}}$ values are directly linked to high SFEs.

Using the derived $M_{\mathrm{H}_{2}}$ values from the $\delta_{\mathrm{GDR}}-Z$ method and the $\mathrm{CO}$ measurements, we can also estimate the $\alpha_{\mathrm{CO}}$ values using the CO-to- $M_{\mathrm{H}_{2}}$ formula:

$$
\mathrm{M}_{\mathrm{H}_{2}}=\alpha_{\mathrm{CO}} \times \mathrm{L}_{\mathrm{CO}}^{\prime} .
$$

The inferred $\alpha_{\mathrm{CO}}$ values for the entire sample range from 6 to 1. [C II $]$ deficient sources have a mean $\left\langle\alpha_{\mathrm{CO}}\right\rangle=1.5$, comparable to that of local ULIRGs. On the other hand, sources that follow the $L_{\mathrm{C} \text { II }}-L_{\mathrm{FIR}}$ relation of Equation (3) have a mean $\left\langle\alpha_{\mathrm{CO}}\right\rangle=5.3$, similar to that of local normal galaxies.

Given the uncertainties coupled with the $M_{\mathrm{H}_{2}}$ estimates, to ensure that the result is not an artefact of the derived $\alpha_{\mathrm{CO}}$ values, in Figure 9 (right), we plot the $L_{\mathrm{C} \text { II }} / L_{\mathrm{FIR}}$ ratio as a function of the direct observables $L_{\mathrm{IR}} / L_{\mathrm{CO}}^{\prime}$. In this plot, we also add a sample of high- $z$ ULIRGs, along with the samples presented in the $L_{\mathrm{CI}} /$ $L_{\mathrm{FIR}}-L_{\mathrm{IR}} / M_{\mathrm{H}_{2}}$ plot. Again, we find that there is a strong anticorrelation between the two ratios, with increasing $L_{\mathrm{IR}} / L_{\mathrm{CO}}^{\prime}$ for decreasing $L_{\mathrm{C} \text { II }} / L_{\mathrm{FIR}}$, at all redshifts and luminosities.

\subsection{The Physical Origin of $C \mathrm{II}$ Emission}

It is worth to attempting to put constraints on its spatial origin, namely, whether it predominantly originates from PDRs or from ionized $\mathrm{H}$ II regions. For this task, ionized nitrogen [N II] $205 \mu \mathrm{m}$

\footnotetext{
28 We assume $M_{\mathrm{HI}} \ll M_{\mathrm{H}_{2}}$, so that $M_{\mathrm{gas}}=M_{\mathrm{H}_{2}}+M_{\mathrm{HI}} \approx M_{\mathrm{H}_{2}}$.

29 The units of $\alpha_{\mathrm{CO}}, M_{\odot} \mathrm{pc}^{-2}\left(\mathrm{~K} \mathrm{~km} \mathrm{~s}^{-1}\right)^{-1}$, are omitted from the text for brevity.
} 


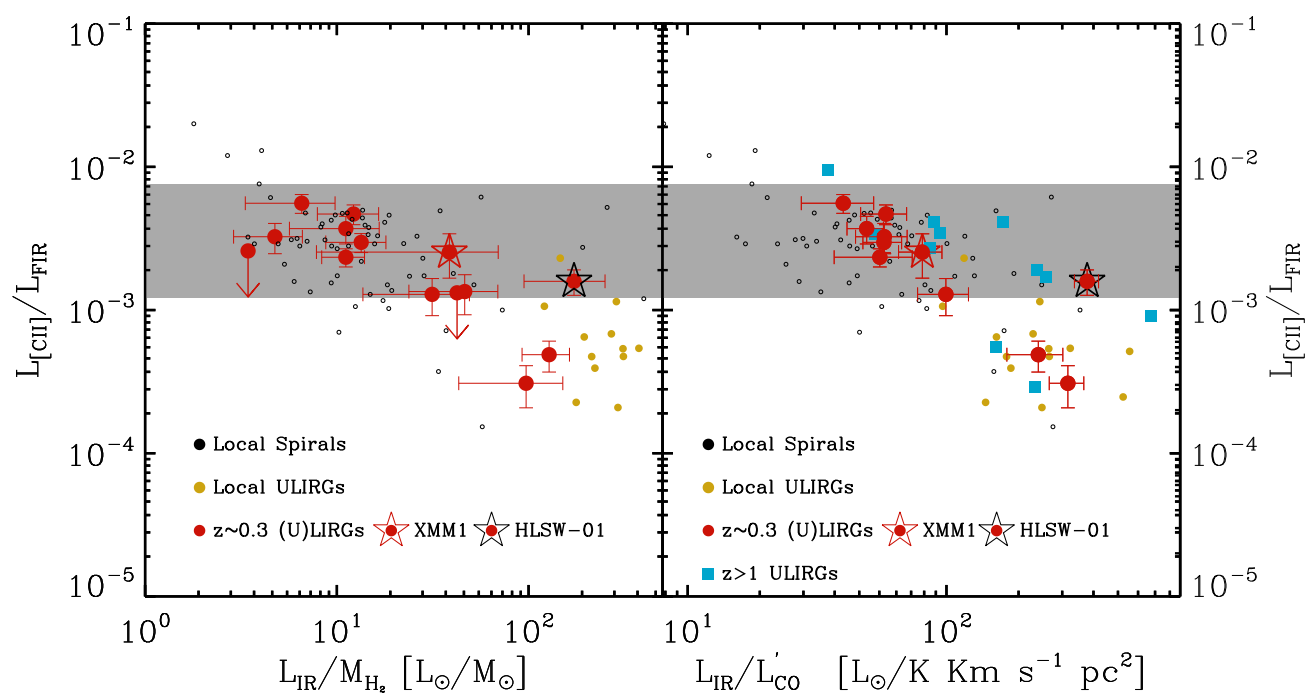

Figure 9. Left: $L_{\mathrm{C} \text { II }} / L_{\mathrm{FIR}}$ vs. $L_{\mathrm{IR}} / M_{\mathrm{H}_{2}}$ for all (U)LIRGs is our sample that have [C II] observations. The vertical shaded region covers the range of $L_{\mathrm{C}} / L_{\mathrm{FIR}}$ values within $1 \sigma$ of Equation (3). Right: $L_{\mathrm{CII}} / L_{\mathrm{FIR}}$ vs. $L_{\mathrm{IR}} / L_{\mathrm{CO}}^{\prime}$ for all (U)LIRGs is our sample that have [C II] and CO observations.

(A color version of this figure is available in the online journal.)

can provide crucial information. With an ionization potential of $14.53 \mathrm{eV}$, slightly above that of hydrogen, [N II] emission traces $\mathrm{H}$ II regions. On the other hand, the ionization potential of carbon is slightly below that of hydrogen, and consequently can arise both from $\mathrm{H}$ II regions and from PDRs. As [N II] and [C II] have very similar critical densities for excitation in ionized gas regions, their line ratio is insensitive to the hardness of the radiation field. Thus, for a given metallicity, their flux ratio can be used as a diagnostic tool to estimate the fraction of carbon emission arising from the $\mathrm{H}$ II regions (where both [C II] and $[\mathrm{N}$ II] are present) versus that arising from the neutral phase (where $\left[\mathrm{N}_{\mathrm{II}}\right]$ is absent). In a $\mathrm{H}$ II region with fixed metallicity, the expected ratio of the two lines is practically independent of the gas density and assuming gas phase abundances of $n([\mathrm{~N} \mathrm{II}]) /$ $n_{\mathrm{e}}=7.9 \times 10^{-5}$ and $n([\mathrm{C} \mathrm{II}]) / n_{\mathrm{e}}=1.4 \times 10^{-4}$ (Savage \& Sembach 1996), we expect a ratio of line luminosities $L_{\text {CII }} /$ $L_{[\mathrm{NII}} \approx 2-3$ in the case where both lines originate from ionized gas regions (Oberst et al. 2006; Decarli et al. 2014).

Since we do not have [N II] detection for any of our sources, we can only place lower limits in the $L_{\mathrm{C} \text { II }} / L_{[\mathrm{N} \text { II] }}$ ratio of our sample by using the $\left[\mathrm{C}_{\mathrm{II}}\right]$ measurements and the $\left[\mathrm{N}_{\mathrm{II}}\right]$ upper limits. While based on the lower limits, we cannot decisively quantify the fraction of $[\mathrm{C} \mathrm{II}]$ emission arising from the PDRs for the majority of the sources the $L_{\mathrm{CII}} / L_{[\mathrm{NII}}$ lower limits are well above the theoretical expectations $\left(L_{\mathrm{CII}} / L_{[\mathrm{NII}}>3.2\right)$ for the case where $\left[\mathrm{C}_{\mathrm{II}}\right]$ emission originates purely from $\mathrm{H}_{\mathrm{II}}$ regions. In particular, it appears that $>50 \%$ of $[\mathrm{C}$ II] emission in intermediate redshift ULIRGs arises from PDRs, similar to high- $z$ ULIRGs (Decarli et al. 2014) and local starbursts (Rigopoulou et al. 2013).

\section{DISCUSSION}

So far we have seen that the far-IR properties of intermediate redshift ULIRGs $(0.2<z<0.8)$ in our sample appear to deviate from those of local ULIRGs, while their overall characteristics resemble those of local normal galaxies and high- $z$ star-forming ULIRGs. In particular, we showed that they exhibit $L_{\mathrm{CI}} / L_{\mathrm{FIR}}$ ratios very similar to that of local spirals and high- $z$ starforming galaxies, with almost an order of magnitude more luminous $[\mathrm{C} \mathrm{II}]$ emission compared to the $[\mathrm{C} \mathrm{II}]$-deficient local
(U)LIRGs. This observation points toward a universal $L_{\mathrm{C}_{\text {II }}}-L_{\mathrm{FIR}}$ relation, with a constant $L_{\mathrm{CII}} / L_{\mathrm{FIR}}$ ratio at all epochs and luminosities (albeit with a considerable scatter), from which at least local ULIRGs and high- $z$, AGN-dominated sources are strong outliers. We have also shown that at all luminosities and redshifts sources follow a tight relation between their farIR color and their fractional [C II] emission $\left(L_{\mathrm{CII}} / L_{\mathrm{FIR}}\right)$. The colder dust temperatures of high- $z$ and intermediate redshift ULIRGs, as found in the current study, offer an explanation for the observed discrepancy between the strength of [C II] emission of local and distant ULIRGs. Furthermore, we have shown that as already reported for local galaxies by GraciáCarpio et al. (2011), among intermediate redshift ULIRGs, sources with higher SFEs tend to exhibit lower $L_{\mathrm{CII}} / L_{\mathrm{FIR}}$ ratios. Having compared the $L_{\mathrm{CII}} / L_{\mathrm{FIR}}$ ratios and dust temperatures in ULIRGs and normal galaxies across cosmic time (using our sample as well as literature data), the next step is to compare their SFEs. Rather than using SFR and gas mass surface densities, we consider the simpler relations between integrated quantities, namely, the total SFR and molecular gas mass or the corresponding observables, $L_{\mathrm{IR}}$ and $L_{\mathrm{CO}}^{\prime}$.

In Figure 10 (left), we compare the CO and IR luminosities of our [C II] sample with other cosmologically relevant galaxy populations: local spirals (COLD GASS; Saintonge et al. 2011), local ULIRGs (Solomon et al. 1997), $z \sim 0.3$ disks (Geach et al. 2011), IRAS-selected $z \sim 0.3$ ULIRGs (Combes et al. 2011), and high- $z$ star-forming galaxies (Daddi et al. 2010a, 2010b; Genzel et al. 2010). The majority of $z \sim 0.3$ (U)LIRGs in our sample follow the $L_{\mathrm{CO}}^{\prime}-L_{\mathrm{IR}}$ relation of normal galaxies from Sargent et al. (2013), which is offset by 0.46 dex with respect to that of local and intermediate redshift IRAS-selected ULIRGs, suggesting that in $z \sim 0.3$, Herschel-selected ULIRGs $\mathrm{CO}$ emission is $\sim 3$ more luminous for a given $L_{\mathrm{IR}}$. Interestingly, the two sources in our sample that deviate the most from the sequence of disks are those with the lowest $L_{\mathrm{CII}} / L_{\mathrm{FIR}}$ ratio. Similarly, in Figure 10 (right), we plot the star formation efficiency $\mathrm{SFE}=L_{\mathrm{IR}} / L_{\mathrm{CO}}^{\prime}$ (in units of $L_{\odot} /\left[\mathrm{K} \mathrm{km} \mathrm{s}^{-1} \mathrm{pc}^{2}\right]$ ) as a function of redshift for the same samples as in the left panel. The ULIRGs in our sample exhibit SFEs comparable to that of high- $z$ star-forming galaxies and local spirals, with two exceptions being the strong outliers of the $L_{\mathrm{CII}}-L_{\mathrm{FIR}}$ relation. 

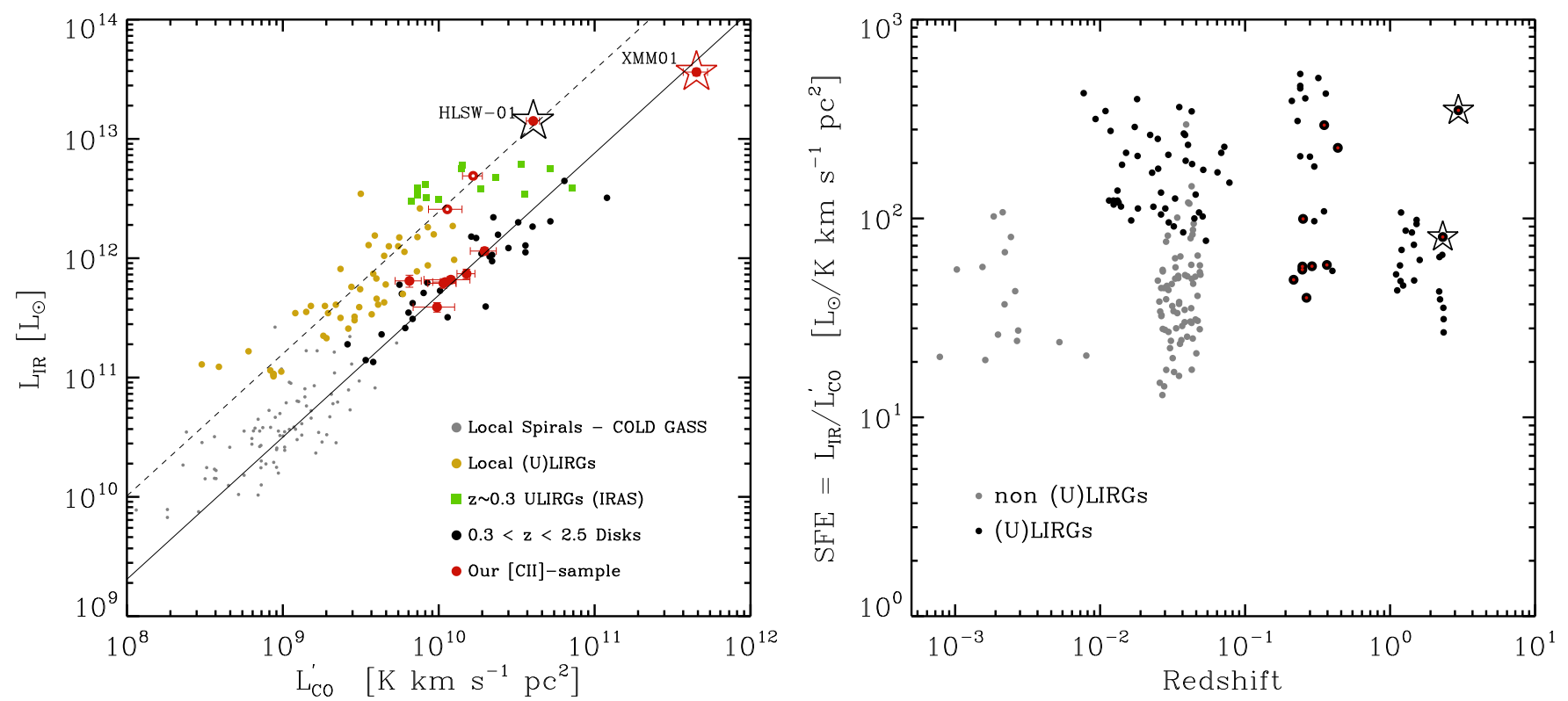

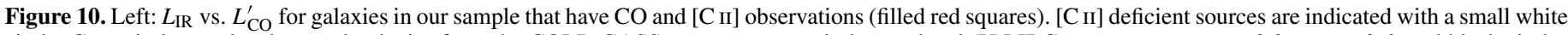

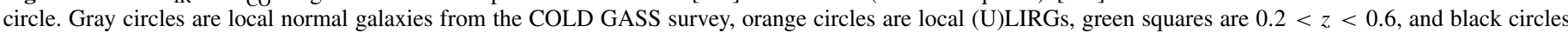

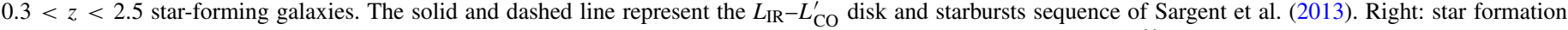

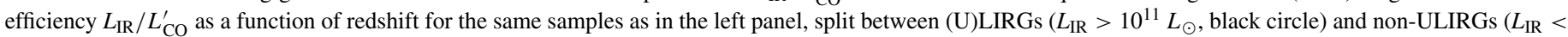
$10^{11} L_{\odot}$, grey circles). Our sample of intermediate redshift (U)LIRGs are indicated with a filled red circle.

(A color version of this figure is available in the online journal.)

In particular, for the entire sample we find a median SFE of $58 L_{\odot} /\left[\mathrm{K} \mathrm{km} \mathrm{s}^{-1} \mathrm{pc}^{2}\right]$ compared to $\sim 300$ for IRAS ULIRGs, $\sim 200$ for local ULIRGs, $\sim 40$ for local spirals, and $\sim 50$ for high- $z$ ULIRG-like disks.

Evidently, Herschel has revealed a population of intermediate redshift ULIRGs with large molecular gas reservoirs that can maintain their high SFRs for prolonged time periods. Indeed, using a typical $\alpha_{\mathrm{CO}}$ value of 4.5 , as derived from the previous analysis, as well as the Kennicutt (1998) formula to convert the $L_{\mathrm{IR}}$ to SFR $\left(\mathrm{SFR}=1 \times 10^{-10} L_{\mathrm{IR}}\right.$ for a Chabrier initial mass function), we find a gas depletion timescale ( $\tau_{\text {dep }}=M_{\mathrm{H}_{2}} /$ SFR) of $\sim 0.8 \mathrm{Gyr}$, a factor of 10 longer than for the shortlived starburst events of local ULIRGs ( $\sim 80 \mathrm{Myr})$. We thus find that the evolution of the star formation activity between a long lasting star formation in a large fraction of high- $z(z>1)$ ULIRG-like star-forming galaxies and a short-lived starburst event in the majority of the local ULIRGs reported in various studies (Genzel et al. 2010; Tacconi et al. 2010; Daddi et al. 2010a; Magdis et al. 2012b) appears to manifest itself already at moderate cosmological times (i.e., 3-4 billion years ago).

An explanation for the observed evolution offered by recent theoretical studies is a shift from a merger induced to more quiescent star formation activity (e.g., Dekel et al. 2009; Davé et al. 2010). Recent morphological and kinematical studies have revealed an increasing number of non-interacting, diskdominated ULIRG-like systems with look-back time (e.g., Förster Schreiber et al. 2009; Kartaltepe et al. 2012; Wang et al. 2012). Also, as discussed above, high- $z$ star-forming galaxies exhibit colder SEDs (e.g., Swinbank et al. 2010; Hwang et al. 2010; Magdis et al. 2010; Symeonidis et al. 2013) compared to local ULIRGs and more extended star formation (e.g., Menéndez-Delmestre et al. 2009; Rujopakarn et al. 2011). Finally, galaxies at all redshifts appear to follow a tight correlation between their star formation SFR and their stellar mass (e.g., Noeske et al. 2007; Elbaz et al. 2007; Pannella et al. 2009; Daddi et al. 2010a; Magdis et al. 2010; Karim et al. 2011). This speaks against stochastic-merger-induced starforming episodes as the main driver of star formation activity, as they would introduce a large scatter in the SFR $-M_{*}$ relation.

Rigopoulou et al. (2014), using the current sample of intermediate redshift ULIRGs, modeled their $L_{\mathrm{CI}} / L_{\mathrm{FIR}}$ and $L_{\mathrm{IR}} / L_{\mathrm{CO}}^{\prime}$ ratios and provided evidence that their star-forming regions are illuminated by moderately intense FUV radiation, with $G_{0}$ in the range $10^{2}-10^{2.5}$ and $G_{0} / \eta \sim 0.1-1 \mathrm{~cm}^{3}$, similar to that of local normal galaxies (e.g., Wolfire et al. 1990) and high- $z$ starforming galaxies (Stacey et al. 2010). On the other hand, local ULIRGs exhibit stronger radiation fields with $G_{0} \sim 10^{2.2-3.6}$ (Farrah et al. 2013). Also, Diaz-Santos et al. (2013) and DíazSantos et al. (2014) recently revealed a clear trend between $L_{\mathrm{C} \text { II }} / L_{\mathrm{FIR}}$ and the compactness of the mid-IR emission of the local galaxies; for a given $L_{\mathrm{IR}}$, sources with lower $L_{\mathrm{C} \text { II }} / L_{\mathrm{FIR}}$ ratios are warmer and their hot dust is confined toward a smaller volume in the center of the galaxy, suggesting more compact star-forming regions. They also found that [C II]-deficient regions in local LIRGs are restricted to the nuclei of the source. The high $L_{\text {C II }} / L_{\text {FIR }}$ ratios of our sample and the colder dust temperature, both indicative of extended star formation activity together with the large molecular gas reservoirs and the long gas depletion timescales, signify a strong evolution in ULIRGs even at moderate redshifts, suggesting that the nature of the ULIRG population changes very fast from exclusively compact-mergerdriven to a more varied population.

The observed steep increase of the molecular gas fraction in star-forming galaxies with look-back time (e.g., Geach et al. 2011; Magdis et al. 2012a, 2012b) can explain both their high SFRs and their long gas depletion timescales, without the need of a merger-induced star-forming episode. Indeed, based on the evolution of gas fraction from Magdis et al. (2012b), galaxies by $z \sim 0.5$ already have two times more molecular gas compared to those in the local universe. While all the above 
provide substantial evidence, a kinematical study of the current sample using IFU spectroscopy with SWIFT (Houghton et al. in preparation) as well high-resolution Hubble Space Telescope imaging that will trace the morphology of the current sample, are best suited to address whether the observed evolution is indeed driven by a change in the triggering mechanism of star formation or by other processes, such an evolution in the galactic scale metal content of ULIRGs with cosmic time.

\section{CONCLUSIONS}

Using Herschel-FTS far-IR spectroscopy, CO observations, and detailed mid-to-far-IR photometry from Spitzer and Herschel, we have preformed a detailed investigation of the properties of 17 Herschel-selected intermediate redshift $(0.2<$ $z<0.8)$ (U)LIRGs. The current sample complements the $T_{\mathrm{d}}$-biased and luminosity-limited IRAS sample of ULIRGs at this redshift range. By combining unique [C II] measurements with molecular gas observations and detailed spectra energy distributions, we reach the following conclusions.

1. The majority of Herschel-selected intermediate redshift ULIRGs have luminous [C $\mathrm{II}$ ] emission lines exhibiting $L_{\mathrm{C} \text { II }} / L_{\mathrm{FIR}}$ ratios that are a factor of 10 higher than that of local ULIRGs and comparable to that of local normal and high- $z$ star-forming galaxies. By combining our sample with data from the literature, we find that the majority of galaxies at all redshift and all luminosities follow a $L_{\mathrm{C}_{\text {II }}}-L_{\mathrm{FIR}}$ relation with a slope of unity and a scatter of 0.3 dex. At least local ULIRGs and high- $z$ AGN-dominated sources are strong outliers from this relation.

2. We find a strong anti-correlation between the $L_{\mathrm{C}} / L_{\mathrm{FIR}}$ ratio and the far-IR color $L_{60} / L_{100}$, in the sense that warmer sources exhibit lower $L_{\mathrm{CI}} / L_{\mathrm{FIR}}$ at all redshifts and all luminosities. Intermediate redshift (U)LIRGs similar to high-z ULIRG like star-forming galaxies exhibit colder dust temperatures.

3. Our sample exhibits lower SFEs compared to local ULIRGs and large molecular gas reservoirs. Sources with higher SFEs tend to have lower $L_{\mathrm{CII}} / L_{\mathrm{FIR}}$ ratios.

4. The high $L_{\mathrm{C} \text { II }} / L_{\mathrm{FIR}}$ ratios, the moderate SFEs $\left(L_{\mathrm{IR}} / L_{\mathrm{CO}}^{\prime}\right.$ or $L_{\mathrm{IR}} / M_{\mathrm{H}_{2}}$ ), and the relatively low dust temperatures (compared to that of local ULIRGs) of our sample of intermediate redshift ULIRGs indicate that the observed evolution of the ULIRG phenomenon between $z=0$ and $z>1$ is already taking place by $z \sim 0.3$.

We warmly thank the referee for constructive comments and suggestions. G.E.M. and D.R. acknowledge support from STFC through grant ST/K00106X/1. G.E.M. acknowledges support from the John Fell Oxford University Press (OUP) Research Fund and the University of Oxford. A.A.-H. acknowledges funding through the Universidad de Cantabria August G. Linares Programme. This work includes observations made with IRAM, which is supported by INSU/CNRS (France), MPG (Germany), and IGN (Spain). Based on observations made with ESO Telescopes at the APEX Observatory under programme ID 090.B-0708A and 091.B-0312A. This paper uses data from Herschel's photometer SPIRE. SPIRE has been developed by a consortium of institutes led by Cardiff University (UK) and including: University of Lethbridge (Canada); NAOC (China); CEA, LAM (France); IFSI, Univ. Padua (Italy); IAC (Spain); Stockholm Observatory (Sweden); Imperial College London,
RAL, UCL-MSSL, UKATC, Univ. Sussex (UK); and Caltech, JPL, NHSC, University of Colorado (USA).

\section{REFERENCES}

Abel, N. P., Dudley, C., Fischer, J., Satyapal, S., \& van Hoof, P. A. M. 2009, ApJ, 701,1147

Aravena, M., Hodge, J. A., Wagg, J., et al. 2014, MNRAS, 442, 558

Armus, L., Charmandaris, V., Bernard-Salas, J., et al. 2007, ApJ, 656, 148

Armus, L., Heckman, T., \& Miley, G. 1987, AJ, 94, 831

Bolatto, A. D., Leroy, A. K., Rosolowsky, E., Walter, F., \& Blitz, L. 2008, ApJ, 686, 948

Bolatto, A. D., Wolfire, M., \& Leroy, A. K. 2013, ARA\&A, 51, 20

Bryant, P. M., \& Scoville, N. Z. 1999, AJ, 117, 2632

Bushouse, H. A., Borne, K. D., Colina, L., et al. 2002, ApJS, 138, 1

Carilli, C. L., Riechers, D., Walter, F., et al. 2013, ApJ, 763, 120

Carter, M., Lazareff, B., Maier, D., et al. 2012, A\&A, 538, A89

Chary, R., \& Elbaz, D. 2001, ApJ, 556, 562

Clements, D. L., Dunne, L., \& Eales, S. 2010, MNRAS, 403, 274

Clements, D. L., Sutherland, W. J., McMahon, R. G., \& Saunders, W. 1996, MNRAS, 279, 477

Combes, F., García-Burillo, S., Braine, J., et al. 2011, A\&A, 528, A124

Conley, A., Cooray, A., Vieira, J. D., et al. 2011, ApJL, 732, L35

Cox, P., Krips, M., Neri, R., et al. 2011, ApJ, 740, 63

Crawford, M. K., Genzel, R., Townes, C. H., \& Watson, D. M. 1985, ApJ, 291, 755

Daddi, E., Bournaud, F., Walter, F., et al. 2010a, ApJ, 713, 686

Daddi, E., Elbaz, D., Walter, F., et al. 2010b, ApJL, 714, L118

Dannerbauer, H., Daddi, E., Riechers, D. A., et al. 2009, ApJL, 698, L178

Dasyra, K. M., Yan, L., Helou, G., et al. 2009, ApJ, 701, 1123

Davé, R., Finlator, K., Oppenheimer, B. D., et al. 2010, MNRAS, 404, 1355

De Breuck, C., Maiolino, R., Caselli, P., et al. 2011, A\&A, 530, L8

Decarli, R., Walter, F., Carilli, C., et al. 2014, ApJL, 782, L17

Dekel, A., Birnboim, Y., Engel, G., et al. 2009, Natur, 457, 451

De Looze, I., Cormier, D., Lebouteiller, V., et al. 2014, A\&A, 568, A62

Desai, V., Armus, L., Spoon, H. W. W., et al. 2007, ApJ, 669, 810

Diaz-Santos, T., Armus, L., Charmandaris, V., et al. 2013, ApJ, 774, 68

Díaz-Santos, T., Armus, L., Charmandaris, V., et al. 2014, ApJL, 788, L17

Donley, J. L., Koekemoer, A. M., Brusa, M., et al. 2012, ApJ, 748, 142

Downes, D., \& Solomon, P. M. 1998, ApJ, 507, 615

Draine, B. T., \& Li, A. 2007, ApJ, 657, 810

Eales, S., Chapin, E. L., Devlin, M. J., et al. 2009, ApJ, 707, 1779

Elbaz, D., Daddi, E., Le Borgne, D., et al. 2007, A\&A, 468, 33

Elbaz, D., Dickinson, M., Hwang, H. S., et al. 2011, A\&A, 533, 119

Farrah, D., Afonso, J., Efstathiou, A., et al. 2003, MNRAS, 343, 585

Farrah, D., Lebouteiller, V., Spoon, H. W. W., et al. 2013, ApJ, 776, 38

Farrah, D., Lonsdale, C. J., Weedman, D. W., et al. 2008, ApJ, 677, 957

Farrah, D., Rowan-Robinson, M., Oliver, S., et al. 2001, MNRAS, 326, 1333

Ferkinhoff, C., Brisbin, D., Parshley, S., et al. 2014, ApJ, 780, 142

Förster Schreiber, N. M., Genzel, R., Bouché, N., et al. 2009, ApJ, 706, 1364

Fu, H., Cooray, A., Feruglio, C., et al. 2013, Natur, 498, 338

Gallerani, S., Neri, R., Maiolino, R., et al. 2012, A\&A, 543, A114

Gao, Y., \& Solomon, P. M. 2004, ApJ, 606, 271

Gavazzi, R., Cooray, A., Conley, A., et al. 2011, ApJ, 738, 125

Geach, J. E., Smail, I., Moran, S. M., et al. 2011, ApJL, 730, L19

Genzel, R., Lutz, D., Sturm, E., et al. 1998, ApJ, 498, 579

Genzel, R., Tacconi, L. J., Gracia-Carpio, J., et al. 2010, MNRAS, 407, 2091

George, R. D., Ivison, R. J., Hopwood, R., et al. 2013, MNRAS, 436, L99

Graciá-Carpio, J., Sturm, E., Hailey-Dunsheath, S., et al. 2011, ApJ, 728, 7

Griffin, M. J., Abergel, A., Abreu, A., et al. 2010, A\&A, 518, L3

Güsten, R., Nyman, L. Å., Schilke, P., et al. 2006, A\&A, 454, L13

Hailey-Dunsheath, S., Nikola, T., \& Stacey, G. J. 2010, ApJ, 714, 162

Houck, J. R., Weedman, D. W., Le Floc'h, E., \& Hao, L. 2007, ApJ, 671, 323

Hwang, H. S., Elbaz, D., Magdis, G., et al. 2010, MNRAS, 409, 75

Iono, D., Yun, M. S., Elvis, M., et al. 2006, ApJL, 645, L97

Ivison, R. J., Smail, I., Papadopoulos, P. P., et al. 2010a, MNRAS, 404, 198 Ivison, R. J., Swinbank, A. M., Swinyard, B.M, et al. 2010b, A\&A, 518, 35

Karim, A., Schinnerer, E., Martínez-Sansigre, A., et al. 2011, ApJ, 730, 61

Kartaltepe, J. S., Dickinson, M., Alexander, D. M., et al. 2012, ApJ, 757, 23

Kaviraj, S., Cohen, S., Windhorst, R. A., et al. 2013, MNRAS, 429, L40

Kennicutt, R. C., Jr. 1998, ARA\&A, 36, 189

La Franca, F., Gruppioni, C., Matute, I., et al. 2004, AJ, 127, 3075

Larson, D., Dunkley, J., Hinshaw, G., et al. 2011, ApJS, 192, 16

Lebouteiller, V., Barry, D. J., Spoon, H. W. W., et al. 2011, ApJS, 196, 8

Le Floc'h, E., Papovich, C., Dole, H., et al. 2005, ApJ, 632, 169

Leroy, A. K., Bolatto, A., Gordon, K., et al. 2011, ApJ, 737, 12 
Lonsdale, C. J., Polletta, M. d. C., Omont, A., et al. 2009, ApJ, 692, 422 Luhman, M. L., Satyapal, S., Fischer, J., et al. 1998, ApJL, 504, L11 Luhman, M. L., Satyapal, S., Fischer, J., et al. 2003, ApJ, 594, 758

Magdis, G. E., Daddi, E., Béthermin, M., et al. 2012a, ApJ, 760, 6

Magdis, G. E., Daddi, E., Elbaz, D., et al. 2011a, ApJL, 740, L15

Magdis, G. E., Daddi, E., Sargent, M., et al. 2012b, ApJL, 758, L9

Magdis, G. E., Elbaz, D., Dickinson, M., et al. 2011b, A\&A, 534, A15

Magdis, G. E., Elbaz, D., Hwang, H. S., et al. 2010, MNRAS, 409, 22

Magdis, G. E., Rigopoulou, D., Helou, G., et al. 2013, A\&A, 558, A136

Magnelli, B., Elbaz, D., Chary, R. R., et al. 2011, A\&A, 528, A35

Malhotra, S., Helou, G., Stacey, G., et al. 1997, ApJL, 491, L27

Malhotra, S., Kaufman, M. J., Hollenbach, D., et al. 2001, ApJ, 561, 766

Meijerink, R., Spaans, M., \& Israel, F. P. 2007, A\&A, 461, 793

Melnick, J., \& Mirabel, I. F. 1990, A\&A, 231, L19

Menéndez-Delmestre, K., Blain, A. W., Smail, I., et al. 2009, ApJ, 699, 667

Moshir, M., Kopman, G., \& Conrow, T. A. O. 1992, in IRAS Faint Source Survey, Explanatory Supplement Version 2, ed. M. Moshir, G. Kopman, \& T. A. O. Conrow (Pasadena, CA: Infrared Processing and Analysis Center, California Institute of Technology)

Mullaney, J. R., Alexander, D. M., Goulding, A. D., \& Hickox, R. C. 2011, MNRAS, 414, 1082

Murphy, E. J., Chary, R.-R., Dickinson, M., et al. 2011, ApJ, 732, 126

Muzzin, A., van Dokkum, P., Kriek, M., et al. 2010, ApJ, 725, 742

Nguyen, H. T., Schulz, B., Levenson, L., et al. 2010, A\&A, 518, L5

Noeske, K. G., Weiner, B. J., Faber, S. M., et al. 2007, ApJL, 660, L43

Norris, R. P., Afonso, J., Appleton, P. N., et al. 2006, AJ, 132, 2409

Oberst, T. E., Parshley, S. C., Stacey, G. J., et al. 2006, ApJL, 652, L125

Oliver, S. J., Bock, J., Altieri, B., et al. 2012, MNRAS, 424, 1614

Oyabu, S., Yun, M. S., Murayama, T., et al. 2005, AJ, 130, 2019

Pannella, M., Carilli, C. L., Daddi, E., et al. 2009, ApJL, 698, L116

Papovich, C., Cool, R., Eisenstein, D., et al. 2006, AJ, 132, 231

Petric, A. O., Armus, L., Howell, J., et al. 2011, ApJ, 730, 28

Pilbratt, G.L, Riedinger, J. R., Passvogel, T., et al. 2010, A\&A, 518, 1

Pope, A., Chary, R.-R., Alexander, D. M., et al. 2008, ApJ, 675, 1171

Pope, A., Scott, D., Dickinson, M., et al. 2006, MNRAS, 370, 1185

Rawle, T. D., Egami, E., Bussmann, R. S., et al. 2014, ApJ, 783, 59

Rawle, T. D., Lucey, J. R., Smith, R. J., \& Head, J. T. C. G. 2013, MNRAS, 433, 2667

Riechers, D. A., Bradford, C. M., Clements, D. L., et al. 2013, Natur, 496, 329

Riechers, D. A., Carilli, C. L., Capak, P. L., et al. 2014, arXiv:1404.7159

Riechers, D. A., Cooray, A., Omont, A., et al. 2011, ApJL, 733, L12

Rigopoulou, D., Hopwood, R., Magdis, G. E., et al. 2014, ApJL, 781, L15
Rigopoulou, D., Hurley, P., \& Swinyard, B. M. 2013, MNRAS, 434, 2015

Rigopoulou, D., Spoon, H. W. W., Genzel, R., et al. 1999, AJ, 118, 2625

Roseboom, I. G., Ivison, R. J., Greve, T. R., et al. 2012, MNRAS, 419, 2758

Roseboom, I. G., Oliver, S. J., Kunz, M., et al. 2010, MNRAS, 409, 48

Rosolowsky, E., Keto, E., Matsushita, S., \& Willner, S. P. 2007, ApJ, 661, 830

Rujopakarn, W., Rieke, G. H., Eisenstein, D. J., \& Juneau, S. 2011, ApJ, 726,93

Saintonge, A., Kauffmann, G., Kramer, C., et al. 2011, MNRAS, 415, 32

Sanders, D. B., \& Mirabel, I. F. 1985, ApJL, 298, L31

Sargent, M. T., Daddi, E., Béthermin, M., et al. 2013, ApJ, 793, 19

Sargsyan, L., Lebouteiller, V., Weedman, D., et al. 2012, ApJ, 755, 171

Savage, B. D., \& Sembach, K. R. 1996, ARA\&A, 34, 279

Scott, K. S., Lupu, R. E., Aguirre, J. E., et al. 2011, ApJ, 733, 29

Sliwa, K., Wilson, C. D., Petitpas, G. R., et al. 2012, ApJ, 753, 46

Smith, J. D. T., Draine, B. T., Dale, D. A., et al. 2007, ApJ, 656, 770

Soifer, B. T., Neugebauer, G., Matthews, K., et al. 2000, AJ, 119, 509

Solomon, P. M., Downes, D., \& Radford, S. J. E. 1992, ApJL, 387, L55

Solomon, P. M., Downes, D., Radford, S. J. E., \& Barrett, J. W. 1997, ApJ, 478, 144

Solomon, P. M., \& Vanden Bout, P. A. 2005, ARA\&A, 43, 677

Spoon, H. W. W., Marshall, J. A., Houck, J. R., et al. 2007, ApJL, 654, L49

Stacey, G. J., Geis, N., Genzel, R., et al. 1991, ApJ, 373, 423

Stacey, G. J., Hailey-Dunsheath, S., Ferkinhoff, C., et al. 2010, ApJ, 724, 957

Swinbank, A. M., Karim, A., Smail, I., et al. 2012, MNRAS, 427, 1066

Swinyard, B. M., Polehampton, E. T., Hopwood, R., et al. 2014, MNRAS, 440, 3658

Swinbank, A. M., Smail, I., Chapman, S. C., et al. 2010, ApJ, 405, 234

Symeonidis, M., Page, M. J., Seymour, N., et al. 2009, MNRAS, 397, 1728

Symeonidis, M., Page, M. J., \& Seymour, N. 2011, MNRAS, 411, 983

Symeonidis, M., Vaccari, M., Berta, S., et al. 2013, MNRAS, 431, 2317

Tacconi, L. J., Genzel, R., Neri, R., et al. 2010, Natur, 463, 781

Tielens, A. G. G. M., \& Hollenbach, D. 1985, ApJ, 291, 722

Valtchanov, I., Virdee, J., Ivison, R. J., et al. 2011, MNRAS, 415, 3473

Vassilev, V., Meledin, D., Lapkin, I., et al. 2008, A\&A, 490, 1157

Venemans, B. P., McMahon, R. G., Walter, F., et al. 2012, ApJL, 751, L25

Voit, G. M. 1992, MNRAS, 258, 841

Wagg, J., Carilli, C. L., Wilner, D. J., et al. 2010, A\&A, 519, L1

Walter, F., Riechers, D., Cox, P., et al. 2009, Natur, 457, 699

Wang, T., Huang, J.-S., Faber, S. M., et al. 2012, ApJ, 752, 134

Weedman, D. W., \& Houck, J. R. 2008, ApJ, 686, 127

Werner, M. W., Roellig, T. L., Low, F. J., et al. 2004, ApJS, 154, 1

Willott, C. J., McLure, R. J., Hibon, P., et al. 2013, AJ, 145, 4

Wolfire, M. G., Tielens, A. G. G. M., \& Hollenbach, D. 1990, ApJ, 358, 116 